\title{
The Barcan formulas and necessary existence: the view from Quarc
}

\author{
Hanoch Ben-Yami ${ }^{1}$ (D)
}

Received: 21 August 2019 / Accepted: 27 June 2020 / Published online: 18 July 2020

(c) The Author(s) 2020

\begin{abstract}
The Modal Predicate Calculus gives rise to issues surrounding the Barcan formulas, their converses, and necessary existence. I examine these issues by means of the Quantified Argument Calculus (Quarc), a recently developed, powerful formal logic system. Quarc is closer in syntax and logical properties to Natural Language than is the Predicate Calculus, a fact that lends additional interest to this examination, as Quarc might offer a better representation of our modal concepts. The validity of the Barcan formulas and their converses is shown by Quarc to be a result of the specific incorporation of quantification in the Predicate Calculus, and not as reflecting a feature of the interaction of quantification and modality more generally. Necessary existence is shown to follow from the identification, in the Predicate Calculus on its canonical interpretation, of particular quantification, ascription of existence and the 'there is' construction, three constructions which are distinguished in both Quarc and Natural Language. The issues surrounding the Barcan formulas, their converses and necessary existence are thus shown to be an artefact of a specific logic system, not an essential feature of our relevant modal concepts or of formal logic.
\end{abstract}

Keywords Modal logic · Quantification · Quantified argument calculus · Barcan formulas · Necessary existence

\section{Introduction}

One might have thought that once we have developed both a modal propositional logic and a non-modal quantified logic, developing a modal quantified logic should be straightforward: simply merge the languages, their rules for formula formation and proof rules, and likewise their semantics. These naïve expectations seem justified: modal concepts are not supposed to change their meaning between propositional

\footnotetext{
$凶$ Hanoch Ben-Yami

benyamih@ceu.edu

1 Department of Philosophy, Central European University, Vienna, Austria
} 
and quantified logic, so the principles capturing it shouldn't be modified when we add quantification to our logic. For the same reason, we shouldn't change the rules expressing quantification when modality is added.-We didn't change the rules for sentential connectives when enriching propositional logic with quantification or with modality, so why should we change those for quantifiers when adding modality, or those for modal operators when adding quantification?

But things do not work so smoothly with the Modal Propositional Calculus and the Predicate Calculus. When the two calculi are merged, the Barcan formulas and their converses, and even more disturbingly necessary existence, follow as theorems. ${ }^{1}$ This is a problem, because it seems that the Natural Language sentences which these formulas purport to translate or at least approximate are not logical truths. It therefore might seem that what is meant in formal logic by existence, possibility and necessity is not what is meant by these concepts in Natural Language.

The formulas and their Natural Language parallels are familiar, so I shall be concise, presenting only what is needed later.

The Barcan formulas (more accurately, schemas) together with their converses state of some quantifiers and modal operators that their scopes can be swapped, or that the de re and de dicto versions of some quantified formulas are equivalent. The Barcan formulas are:

1. $\diamond \exists x \phi(x) \rightarrow \exists x \diamond \phi(x)$

2. $\forall \mathrm{x} \square \phi(\mathrm{x}) \rightarrow \square \forall \mathrm{x} \phi(\mathrm{x})$

And their converses:

3. $\exists \mathrm{x} \diamond \phi(\mathrm{x}) \rightarrow \diamond \exists \mathrm{x} \phi(\mathrm{x})$

4. $\square \forall \mathrm{x} \phi(\mathrm{x}) \rightarrow \forall \mathrm{x} \square \phi(\mathrm{x})$

In each pair, the two formulas are equivalent, so I shall discuss only one of them at this place, the existential version.

Suppose the maximal height of humans is determined, among other things, by their genetic makeup, which we shall assume, for the sake of argument, is essential to them, and that some rare sperms and ova can bring about a human taller than three metres. The following de dicto statement is then true:

5. It is possible that there be a person over three metres tall.

Suppose, however, that none of the people who has been or shall be born developed from such a sperm and ovum. The following, de re statement, is then apparently false:

6. There's someone (or something) who could be a person over three meters tall.

(If (5) or (6) is ambiguous and allows both a de dicto and a de re reading, then when read with the meaning intended here, the former is true while the latter false.) Sentences (5) or (6) are often translated, respectively, by the antecedent and consequent of (1), so apparently, Natural Language sentences translated by the Barcan formula (1) can be false.

\footnotetext{
1 (Prior 1956) contains the first proof of the Barcan formulas. The proofs of the converse Barcan formulas and of necessary existence are comparatively simple.
} 
Next, let us interpret the formula $\phi(\mathrm{x})$ as meaning, $x$ does not exist. The following de re statement is then true:

7. There is someone (or something) who might not have existed.

However, the de dicto parallel seems false:

8. It is possible that there be someone who doesn't exist.

With (7) translated by the antecedent of (3) and (8) by its consequent, a Natural Language sentence translated by the converse Barcan formula (3) can, apparently, be false as well.

Those familiar with the possibilist versus actualist debate (see Garson 2016, Menzel 2018) might now be thinking of ways found in the literature to either eliminate or justify this apparent discrepancy. I shall not consider these at this place, as I am only introducing the issues. I address the debate in the last section of the paper.

The standard interpretation of the existential quantifier in the Predicate Calculus is as expressing existence. That $s$ exists can be formalised by,

9. $\exists x(x=s)$

And that $s$ necessarily exists by,

10. $\square \exists x(\mathrm{x}=\mathrm{s})$

These are theorems of the calculus, resulting from the merge we are considering, as well as the following formal rendering of Everything necessarily exists:

\section{1. $\forall \mathrm{y} \square \exists \mathrm{x}(\mathrm{x}=\mathrm{y})$}

It seems that this is clearly wrong for our Natural Language concept of existence: Isaac existed, but if Abraham and Sarah hadn't met, he wouldn't have.

These undesirable results have made philosophers and logicians attempt to develop quantified modal systems in which the above formulas are not theorems, and Kripke and others have succeeded in devising some such systems. However, the modifications needed to block the Barcan formulas, their converses and necessary existence have seemed ad hoc, and in addition resulted in more cumbersome formal systems (Williamson 2013; Garson 2016). Consequently, they have not been generally accepted as providing an improved representation of our modal concepts. Following this, some have welcomed these formulas as discoveries, carrying a metaphysical lesson: for instance, everything that exists indeed exists necessarily; Socrates, when he apparently ceased to exist, in fact started existing as an abstract object (Williamson 2013). Most philosophers have not found this last reaction convincing.

In this paper, I suggest a different way to assess these results. My claim will be that the Barcan formulas, their converses and necessary existence are artefacts of the specific formal quantified logic system we have been using, namely, the Predicate Calculus. I shall present an alternative quantified formal logic, the Quantified Argument Calculus (Quarc), in which parallel issues do not arise. I shall also use Quarc to shed light on the specific conception of quantification of the Predicate Calculus and on its standard semantics, showing both to be merely optional and, arguably, to misrepresent concepts of Natural Language they are often taken to represent. 
Quarc is a powerful formal logic system, first introduced in Ben-Yami's "The Quantified Argument Calculus" (2014), based on work published by Ben-Yami over the preceding decade, primarily (Ben-Yami 2004), and closely related to the calculus found in Lanzet and Ben-Yami (2004). It is closer in syntax to Natural Language than is the Predicate Calculus, sheds light on the logical role of some of Natural Language's features which it incorporates (such as copular structure, converse relation terms and anaphora), and is also closer to Natural Language in the logical relations it validates. (Ben-Yami 2014) contains a natural deduction system for Quarc and a truthvaluational, substitutional semantics; this system has been shown to be sound and complete (Ben-Yami 2014; Ben-Yami and Pavlovic, unpublished). Quarc has since been extended into a sound and complete three-valued system with defining clauses, using model-theoretic semantics (Lanzet 2017). In this latter version it was shown to contain a semantically isomorphic image of the Predicate Calculus. Thus, Quarc has been shown to be at least as strong as the first-order Predicate Calculus, and moreover, the proofs in these works shed light on the nature of quantification in the Predicate Calculus (see there and below for details). In other works (Pavlovic 2017; Pavlovic and Gratzl 2019a, b), a sequent calculus has been developed for several versions of Quarc and various properties of the system, such as cut-elimination, subformula property, consistency and others, were proved. Quarc has also been used to investigate Aristotelian logic, both assertoric and modal, in works mentioned above as well as in Raab (2018).

Quarc is introduced in the next section of this paper. As shall be seen there, it is closer to Natural Language than is the Predicate Calculus in its syntax, in the features it incorporates, and in the logical relations it preserves (this has been further explored, vis-à-vis Moss's Natural Logic, in Ben-Yami forthcoming). It thus has a good claim to represent the logic of Natural Language better than does the Predicate Calculus. I also show there how quantification in the Predicate Calculus can be represented in Quarc, and address their comparative powers.

I then extend Quarc to incorporate modality (Sect. 3). Having done that, I use Quarc to analyse the Barcan formulas and their converses (Sect. 4). I end by applying Quarc to the analysis of the necessary existence issue (Sect. 5).

As we shall see, the merging of Quarc with Modal Propositional Logic does not give rise to issues paralleling those mentioned above. Quarc also helps understand why these issues do arise when the Predicate Calculus is merged with Modal Propositional Logic. This provides us with reasons to analyse or model quantification in Natural Language with Quarc rather than with the Predicate Calculus, reasons additional to those already provided in earlier works. The naïve expectations mentioned above, that merging modal propositional logic with quantified logic should be quite straightforward, turn out to be justified if we use Quarc rather than the Predicate Calculus.

Before we proceed, a comment about my claims regarding Natural Language. There are several thousands of languages used or documented around the world, and collecting them all under a capitalised 'Natural Language' requires empirical justification. Obviously, I did not carry out myself the required investigation, although I did sample quite a few language families. (By consulting native speakers, I have checked from each of at least the following families one or often quite a few languages: Afro-Asiatic, Indo-European, Uralic, Turkic, Sino-Tibetan, Koreanic, Japonic, Austroasiatic, Niger- 
Congo.) My claims also rely on empirical research carried out by linguists, primarily as reported and referenced in Peters and Westerståhl (2006) and McNally (2011) (see also references below), but as communicated in discussions as well. These grounds do leave open the possibility that some natural language be found which incorporates quantification in a way quite unlike Quarc's, or which unlike it has no modes of predication, say, and Quarc would then fail to represent it adequately. For various reasons, empirical as well as theoretical, I don't find a wide divergence likely; yet this is not the place to discuss them. Accordingly, my claims below about capitalised Natural Language should be taken as only partly empirically supported, and further evidence for or against them would be needed, and appreciated.

\section{The quantified argument calculus}

By now, Quarc has been presented in several publications (Ben-Yami 2014; Lanzet 2017; Pavlovic 2017; Pavlovic and Gratzl 2019a, b). There is therefore no need for an additional full exposition. Moreover, for our purposes below, we do not need to employ the full version of Quarc that was introduced in Ben-Yami (2014). Accordingly, although I shall first informally introduce the full Quarc language of that paper, the subsequent formal introduction shall be of a reduced version (but with the addition of identity), one which we will then continue to use.

\subsection{Informal introduction of the system}

Consider a simple subject-predicate or argument-predicate sentence:

12. Alice is polite

Its grammatical form can be represented by

13. (Alice) is polite

with the argument in parenthesis, followed by the copula and then the predicate. In the Predicate Calculus, we formalise this sentence by

14. $\mathrm{P}(\mathrm{a})$

Quarc does not deviate from this formalisation, apart from a typographical change: the arguments, in Quarc, are written to the left of the predicate:

15. (a)P

Similarly,

16. Alice loves Bob

is formalised, in Quarc, by

17. $(\mathrm{a}, \mathrm{b}) \mathrm{L}$

Consider now the quantified sentence,

18. Every student is polite 
Its grammatical form can be represented by

19. (Every student) is polite

Here, grammatically, the argument is the noun phrase 'every student'. In it, the quantifier 'every' attaches to the unary predicate 'student', and together they form a quantified argument. This is the way quantification is incorporated in Quarc:

20. $(\forall S) P$

Namely, quantifiers are not sentential operators. Rather, they attach to unary predicates to form quantified arguments. Some other examples:

21. Some students are polite

22. Every girl loves Bob

23. Every girl loves some boy

are formalised (respectively; likewise below) by,

24. $(\exists S) P$

25. $(\forall \mathrm{G}, \mathrm{b}) \mathrm{L}$

26. $(\forall \mathrm{G}, \exists \mathrm{B}) \mathrm{L}$

This basic departure in the treatment of quantification requires a few additional ones. One such is the need to reintroduce the copular structure and, with it, modes of predication, as in Aristotelian logic. In Natural Language, we can negate sentence (12), 'Alice is polite', in two ways:

27. It's not the case that Alice is polite

28. Alice isn't polite

The Predicate Calculus allows only the first mode of negation-the one rarer and somewhat artificial in Natural Language-namely, sentential negation. Quarc, however, also allows the negation symbol to be written between the argument or arguments and the predicate, signifying negative predication, by contrast to affirmative one. These two sentences are thus formalised by

29. $\neg((\mathrm{a}) \mathrm{P})$

30. (a) $\neg P$

Parentheses can be omitted without ambiguity in these formulas, and they can be written as $\neg \mathrm{aP}$ and $\mathrm{a} \neg \mathrm{P}$. Since the argument is singular, these two formulas are equivalent, and they shall be defined as such both in the proof system and in the semantics below. However, the equivalence does not hold when the argument is quantified:

31. It's not the case that some students are polite

32. Some students aren't polite

formalised by:

33. $\neg(\exists \mathrm{SP})$

34. $(\exists S) \neg P$ 
These formulas will not be equivalent either in the proof system or in the semantics.

All natural languages have the means of reordering the noun-phrases in relational sentences without changing, if the arguments are all singular, what is said by the sentences. Different languages achieve this by different means. English often accomplishes it by changing from active- to passive-voice:

\section{Alice loves Bob}

36. Bob is loved by Alice

In the singular case, the two are logically equivalent. But again, this is not generally the case when the arguments are quantified:

37. Every girl loves some boy

38. Some boy is loved by every girl

(Even if these sentences are ambiguous as to the order of quantifier scope, each sentence's prominent reading differs from the other's.) Quarc incorporates this reordering by having an $n$-ary predicate written with a permutation of the $1,2, \ldots \mathrm{n}$ sequence as superscripts to its right. Sentences (35) to (38) are then formalised by,

39. $(\mathrm{a}, \mathrm{b}) \mathrm{L}$

40. $(\mathrm{b}, \mathrm{a}) \mathrm{L}^{2,1}$

41. $(\forall \mathrm{G}, \exists \mathrm{B}) \mathrm{L}$

42. $(\exists B, \forall G) \mathrm{L}^{2,1}$

As with negation, formulas with only singular arguments are defined as equivalent in both proof system and semantics, while this equivalence will not generally hold for sentences with quantified arguments.

The last additional feature of Quarc is its use of anaphora. Consider the two sentences,

43. Bob loves Bob

44. Bob loves himself

The former is rarely used, although one of its uses is to explain the use of the reflexive pronoun 'himself' in the latter. The reflexive pronoun 'himself' in (44) is anaphoric on the earlier occurrence of 'Bob', its source, in the sense that it can be replaced by its source and the sentence will still say the same thing. This replaceable anaphor is what Geach called, a pronoun of laziness (1962, Sect. 76). Quarc incorporates it by using a Greek letter for the anaphor, written as a subscript to the right of its source. Accordingly, it formalises (43) and (44) by:

45. $(\mathrm{j}, \mathrm{j}) \mathrm{L}$

46. $\left(\mathrm{j}_{\alpha}, \alpha\right) \mathrm{L}$

The formalisation of quantified sentences in which quantified arguments have anaphors is similar:

47. Every man loves himself

48. $\left(\forall \mathrm{M}_{\alpha}, \alpha\right) \mathrm{L}$

As with negation and reordering, if all arguments are singular, then a Quarc formula with an anaphor and the formula with that anaphor replaced by its source are defined 
as equivalent in both proof system and semantics. However, the anaphor is no longer replaceable by its source when the latter is quantified, neither in Natural Language nor in Quarc.

With this I conclude the informal introduction of Quarc and turn to the more rigorous introduction of the formal system. However, for the purposes of the discussion below, we don't need to use formulas with either reordered predicates or anaphora. I therefore introduce a reduced version of Quarc, which will make it easier to follow and focus on the main argument of this paper. The interested reader is referred to the works mentioned above to see how these are incorporated in the full version of Quarc.

\subsection{Vocabulary of Quarc}

The language $L$ of Quarc contains the following symbols:

- Predicates: P, Q, R,.., denumerably many and with a fixed arity, including the binary predicate $=$.

- Singular arguments (SAs): a, b, c,..., denumerably many.

- Sentential operators: $\neg, \wedge, \vee, \rightarrow, \leftrightarrow$.

- Quantifiers: $\forall, \exists$.

- Comma, parentheses.

If $\mathrm{P}$ is a unary predicate, then $\forall \mathrm{P}$ and $\exists \mathrm{P}$ will be called, quantified arguments (QAs). An argument is a singular argument or a quantified one. The quantifier $\exists$ is called, the particular quantifier, not the existential, and is read some, not there is; the reasons for that are discussed in Sect. 5.

\subsection{Formulas of Quarc}

The following rules specify all the ways in which formulas can be generated.

- (Basic formula) If $\mathrm{P}$ is an $n$-ary predicate and $\mathrm{c}_{1}, \ldots, \mathrm{c}_{\mathrm{n}} \mathrm{SAs}$, then $\left(\mathrm{c}_{1}, \ldots, \mathrm{c}_{\mathrm{n}}\right) \mathrm{P}$ is a formula, called a basic formula.

- (Negative predication) If $\mathrm{P}$ is an $n$-ary predicate and $\mathrm{c}_{1}, \ldots, \mathrm{c}_{\mathrm{n}} \mathrm{SAs}$, then $\left(\mathrm{c}_{1}, \ldots, \mathrm{c}_{\mathrm{n}}\right) \neg \mathrm{P}$ is a formula.

- (Identity) If $c_{1}$ and $c_{2}$ are SAs then $c_{1}=c_{2}$ is a formula. $c_{1}=c_{2}$ is an alternative way of writing $\left(\mathrm{c}_{1}, \mathrm{c}_{2}\right)=$.

- (Sentential operators) If $\phi$ and $\psi$ are formulas, so are $\neg(\phi),(\phi) \wedge(\psi),(\phi) \vee(\psi)$, $(\phi) \rightarrow(\psi)$ and $(\phi) \leftrightarrow(\psi)$. The parentheses surrounding formulas are called sentential parentheses. They can be omitted if no ambiguity arises.

- (Quantification) If $\phi$ is a formula containing an occurrence of an SA c, and substituting the quantified argument $\mathrm{qP}$ for $\mathrm{c}$ will result in $\mathrm{qP}$ governing $\phi$ (see definition below), then $\phi(\mathrm{qP} / \mathrm{c})$ is a formula. $(\phi(\mathrm{qP} / \mathrm{c})$ is the formula in which $\mathrm{qP}$ replaced the occurrence of c.) 


\subsubsection{Governance}

The notion of governance, which is related to that of scope in the Predicate Calculus, is defined as follows:

(Governance) An occurrence qP of a QA governs a string of symbols A just in case $\mathrm{qP}$ is the leftmost $\mathrm{QA}$ in $\mathrm{A}$ and $\mathrm{A}$ does not contain any other string of symbols, (B), in which the displayed parentheses are a pair of sentential parentheses, such that (B) contains $\mathrm{qP}$.

Once anaphors are introduced, the notion of governance becomes non-trivial and its definition needs elaboration. Since they are not introduced in this formal part, determining whether a quantified argument governs a formula is straightforward. For instance, $\exists \mathrm{S}$ governs the formulas $(\exists \mathrm{S}) \mathrm{P},(\exists \mathrm{S}) \neg \mathrm{P},(\mathrm{a}, \exists \mathrm{S}) \mathrm{L}$ and $(\exists \mathrm{S}, \forall \mathrm{P}) \mathrm{L}$ - the last one because it is to the left of $\forall \mathrm{P}$. By contrast, $\exists \mathrm{S}$ does not govern $\neg((\exists S) \mathrm{P})$, since it is contained in $((\exists \mathrm{S}) \mathrm{P})$; nor $((\exists \mathrm{S}) \mathrm{P}) \wedge(\mathrm{aQ})$, as it is contained in $((\exists \mathrm{S}) \mathrm{P})$; nor $(\forall \mathrm{Q}, \exists \mathrm{S}) \mathrm{L}$, since $\forall Q$ is to its left. For the reduced Quarc language of this paper, a simpler definition of governance could be provided, practically listing the schemas of formulas governed by a QA; I preferred to use this definition in order to facilitate the transition to fuller Quarc languages.

\subsection{Model-theoretic semantics}

Although (Ben-Yami 2014) uses truth-valuational semantics for Quarc, I use here model-theoretic semantics. The points made in the following sections are valid on both approaches, and the greater familiarity with model-theoretic semantics will not divert attention from them. The use of model-theoretic semantics will also block the otherwise possible suspicion, that the results obtained below might be due to the nonstandard semantics: the different formal system can establish these results on either semantic approach.

Model-theoretic semantics was used in Lanzet (2017) for a richer, three-valued version of Quarc. The semantics below mainly follows that paper's, adapted to a two-valued, reduced version of Quarc.

(Interpretation) An interpretation for $L$ is a function $M$ for which the following conditions hold (where $X^{M}$ is the image of $X$ under $M$ ):

- The domain of the function $M$ is the set of all singular arguments and predicates of $L$, apart from $=$.

- $\quad$ For every $n$-ary predicate $\mathrm{R}$ in $L, \mathrm{R}^{M}$ is a set of $n$-tuples.

- $\quad$ For any unary predicate $\mathrm{P}$ in $L, \mathrm{P}^{M}$ is non-empty.

Notice that an interpretation in Quarc has no domain of quantification but is simply an interpretation function. See (Ben-Yami 2004, pp. 59-60) and (Lanzet 2017), especially Sect. 2, on this. Consequently, in Quarc, the model is the interpretation; it is not an ordered pair of a domain and an interpretation function. The third, non-emptiness condition above is introduced here to simplify the semantics. It can be eliminated, as was done for instance in Lanzet (2017), which then developed a three-valued semantics 
for the calculus (see also below). This would have made the semantics more natural but would also have added unnecessary complexity (as any other choice would also have), given our purposes in this paper, and is therefore not done here.

(Truth-value in an interpretation) We define inductively the truth-value $[\phi]^{M}$ of any formula $\phi$ in an interpretation $M$. The truth-value $[\phi]^{M}$ will always be either $\mathbf{T}$ (true) or $\mathbf{F}$ (false).

- (Basic formulas)

$\left[\left(\mathrm{c}_{1}, \ldots, \mathrm{c}_{n}\right) \mathrm{P}\right]^{M}=\mathbf{T}$ if $<\mathrm{c}_{1}^{M}, \ldots, \mathrm{c}_{n}^{M}>\in \mathrm{P}^{M}, \mathbf{F}$ otherwise.

$\left[\mathrm{c}_{1}=\mathrm{c}_{2}\right]^{M}=\mathbf{T}$ if $\mathrm{c}_{1}^{M}$ is $\mathrm{c}_{2}^{M}, \mathbf{F}$ otherwise.

$\mathrm{c}_{i}$ here are any singular arguments, $n \geq 1$, and $\mathrm{P}$ any $n$-ary predicate.

- (Sentential operators) These definitions are standard. For instance,

$[\neg \phi]^{M}=\mathbf{T}$ if $[\phi]^{M}=\mathbf{F}, \mathbf{F}$ otherwise.

- (Negative predication)

$$
\left[\left(\mathrm{c}_{1}, \ldots, \mathrm{c}_{n}\right) \neg \mathrm{P}\right]^{M}=\left[\neg\left(\mathrm{c}_{1}, \ldots, \mathrm{c}_{n}\right) \mathrm{P}\right]^{M}
$$

To define the truth conditions of quantified formulas, we rely on the following two definitions:

- $\quad(\alpha \mid c \text {-variant })^{2}$ For any object $\alpha$, any interpretation $M$ and any singular argument $\mathrm{c}$, the $\alpha \mid \mathrm{c}$-variant of $M$, or $M_{\alpha \mid \mathrm{c}}$, is the interpretation that sends $\mathrm{c}$ to $\alpha$ and coincides with $M$ on any other input.

- (True of/false of) If $\phi(\mathrm{c})$ is a formula and $\phi(\mathrm{x})$ is the expression $\phi(\mathrm{x} / \mathrm{c})$, in which $\mathrm{x}$ replaced all occurrences of $\mathrm{c}$, then $\phi(\mathrm{x})$ is true of (false of) $\alpha$ in $M$ if $\phi(\mathrm{c})$ is true (false) in $M_{\alpha \mid c}$.

In the above definition, $\phi(\mathrm{x})$ is not a formula of Quarc, which does not include $\mathrm{x}$ in its vocabulary. Such strings of symbols are auxiliaries for determining truth-values of formulas. We can now define the truth-value in an interpretation of quantified formulas:

- $\quad$ (Quantification) If $\mathrm{P}$ is any unary predicate, $\mathrm{q}$ is a quantifier, $\phi(\mathrm{qP})$ a formula governed by $\mathrm{qP}$ and $\phi(\mathrm{x})$ is $\phi(\mathrm{x} / \mathrm{qP})$, in which $\mathrm{x}$ replaced the governing occurrence of $\mathrm{qP}$, then:

$[\phi(\forall \mathrm{P})]^{M}=\mathbf{T}$ if $\phi(\mathrm{x})$ is true in $M$ of every element of $\mathrm{P}^{M}, \mathbf{F}$ otherwise.

$[\phi(\exists \mathrm{P})]^{M}=\mathbf{T}$ if $\phi(\mathrm{x})$ is true in $M$ of some element of $\mathrm{P}^{M}, \mathbf{F}$ otherwise.

Notice that quantification in $\phi(\forall \mathrm{P})$ and $\phi(\exists \mathrm{P})$ is over the elements of $\mathrm{P}^{M}$, not over any domain which is unspecified by the formulas.

If the non-emptiness condition of (Interpretation) is not imposed, that is, if unary predicates can have empty extension, (Quantification) should also specify the truth-values of $\phi(\forall \mathrm{P})$ and $\phi(\exists \mathrm{P})$ in case $\mathrm{P}^{M}$ is the empty set. The option I find most natural and which was also adopted in Lanzet (2017) is to make both $[\phi(\forall \mathrm{P})]^{M}$ and $[\phi(\exists \mathrm{P})]^{M}$ 'undefined', $\mathbf{U}$, in this case and develop a three-valued system (Other choices can also be made-see (Pavlovic and Gratzl 2019a, b)). If this option is adopted, then instead of $(\forall \mathrm{P}) \mathrm{P}$ and $(\exists \mathrm{P}) \mathrm{P}$ being $\mathbf{T}$ on every model, they shall be on

\footnotetext{
$2 \alpha$ was used for anaphors in the informal introduction of Quarc. As anaphora is not used in this paper apart from the two formulas provided there, I shall use $\alpha$ hereafter only as specified in these and later definitions, for items in the range of predicates.
} 
each model either both T or both U. (Notice that there's no difference in this respect between $\forall$ and $\exists$.) However, as was said above, we shall impose the non-emptiness condition in this paper, as the additional complexity resulting from its elimination does not contribute to the issues on which we focus.

The definitions of models and entailment are standard:

$M$ is a model of a formula $\phi$ iff $[\phi]^{M}=\mathbf{T}$; it is a model of a set of formulas iff it is a model of each formula of the set.

(Entailment) A set $\Gamma$ of formulas entails a formula $\phi$, or the argument with $\Gamma$ as premises and $\phi$ as conclusion is valid, or $\Gamma \vDash \phi$, iff every model of $\Gamma$ is a model of $\phi$.

As an example, let us show that $(\forall \mathrm{S}) \mathrm{P} \vDash(\exists \mathrm{P}) \mathrm{S}$, i.e., that Every $S$ is $P$ entails Some $P$ are $S$, an Aristotelian conversion whose standard translation into the Predicate Calculus is invalid. Intuitively (Euler or Venn diagrams can be used here), (1) a model of Every $S$ is $P$ is one in which every member of $\mathrm{S}$ is also a member of $\mathrm{P}$; and (2) a model of Some $P$ are $S$ is such in which there is an element which is both in $\mathrm{P}$ and in S. If Every $S$ is $P$ is true then there is at least one member of $S$ - here Quarc departs from the way in which the Predicate Calculus analyses universal quantification-which from (1) is also a member of P, and so, from (2), both sentences are true. And formally: If $M$ is a model of $(\forall \mathrm{S}) \mathrm{P}$ then, according to (Quantification), (x)P is true in $M$ of all the elements of $\mathrm{S}^{M}$, and this presupposes that $\mathrm{S}^{M}$ has elements. Let us consider one such element, say $\alpha \in \mathrm{S}^{M}$. According to (Basic formulas), since (x)P is true of $\alpha, \alpha \in \mathrm{P}^{M}$. But then, (x)S is also true of some element of $\mathrm{P}^{M}$, namely $\alpha$, and therefore $(\exists \mathrm{P}) \mathrm{S}$ is true in $M$. QED. (Notice that this entailment holds even if the non-emptiness condition is removed and the undefined option suggested above is adopted.)

\subsection{Proof system}

The proof system used here is based on that found in Ben-Yami (2014) and Ben-Yami and Pavlovic (unpublished), with the omission of the rules for reordered forms and anaphora. I use a Lemmon-style natural deduction system, based on the one introduced by Jaśkowski (1934) and further developed and streamlined by Fitch (1952), Lemmon (1978) and others. Proofs are written as follows:

(Proof) A proof is a sequence of lines of the form $<\mathrm{L}$, (i), $\phi, \mathrm{R}>$, where $\mathrm{L}$ is a possibly empty list of line numbers; (i) the line number in parentheses; $\phi$ a formula; and $\mathrm{R}$ the justification, a name of a derivation rule possibly followed by line numbers, written according to one of the derivation rules specified below. $\phi$ is said to depend on the formulas listed in L. The line numbers in L are written without repetitions and in ascending order. The formula in the last line of the proof is its conclusion. If there is a proof with the formula $\phi$ as conclusion, depending only on formulas from the set $\Gamma$, then $\phi$ is provable from $\Gamma$, or $\Gamma \vdash \phi$.

I next list the derivation rules of the system. 


\section{(Derivation rules)}

- (Premise) As any line of a proof, any formula can be written, depending on itself, its justification being Premise:

i

(i)

$\phi$

Premise

- (Identity Introduction, = I) As any line of the proof a formula of the form $\mathrm{c}=\mathrm{c}$ can be written, depending on no premises, with its justification being $=\mathrm{I}$.

\begin{tabular}{llll}
\hline- & (i) & $\mathrm{c}=\mathrm{c}$ & $=\mathrm{I}$ \\
\hline
\end{tabular}

The dash, -, which is not part of the language, is used here and below to emphasise that a formula does not depend on any premise, i.e., that it is a theorem.

- (Identity Elimination, = E) (This and the following rules specify how to add a line to a proof which contains preceding lines of the specified forms.) Let $\phi$ be a basic formula containing occurrences $\mathrm{t}_{1}, \ldots, \mathrm{t}_{n}$ of the singular argument $\mathrm{t}(\phi$ may also contain additional occurrences of $\mathrm{t}$ ).

\begin{tabular}{llll}
\hline $\mathrm{L}_{1}$ & (i) & $\phi$ & \\
$\mathrm{L}_{2}$ & (j) & $\mathrm{t}=\mathrm{c}$ & \\
$\mathrm{L}_{1}, \mathrm{~L}_{2}$ & (k) & $\phi\left(\mathrm{c} / \mathrm{t}_{1}, \ldots, \mathrm{c} / \mathrm{t}_{n}\right)$ & $=\mathrm{E} \mathrm{i}, \mathrm{j}$ \\
\hline
\end{tabular}

Where ' $\mathrm{L}_{1}, \mathrm{~L}_{2}$ ' is the list of numbers occurring either in $\mathrm{L}_{1}$ or in $\mathrm{L}_{2}$.

- (Propositional Calculus Rules, PCR) We allow the usual derivation rules of the Propositional Calculus.

- (Sentence negation to Predication negation, SP) Let P be an $n$-ary predicate and $\mathrm{c}_{1}$, $\ldots, \mathrm{c}_{n}$ singular arguments.

\begin{tabular}{llll}
\hline $\mathrm{L}$ & (i) & $\neg\left(\mathrm{c}_{1}, \ldots, \mathrm{c}_{n}\right) \mathrm{P}$ & \\
$\mathrm{L}$ & (j) & $\left(\mathrm{c}_{1}, \ldots, \mathrm{c}_{n}\right) \neg \mathrm{P}$ & $\mathrm{SP} \mathrm{i}$ \\
\hline
\end{tabular}

- (Predication negation to Sentence negation, PS) Let $\mathrm{P}$ be an $n$-ary predicate and $\mathrm{c}_{1}$, $\ldots, \mathrm{c}_{n}$ singular arguments.

\begin{tabular}{llll}
\hline $\mathrm{L}$ & (i) & $\left(\mathrm{c}_{1}, \ldots, \mathrm{c}_{n}\right) \neg \mathrm{P}$ & \\
$\mathrm{L}$ & (j) & $\neg\left(\mathrm{c}_{1}, \ldots, \mathrm{c}_{n}\right) \mathrm{P}$ & $\mathrm{PS} \mathrm{i}$ \\
\hline
\end{tabular}

- (Universal Introduction, $\forall \mathrm{I})$ Let $\phi(\forall \mathrm{P})$ be a formula governed by $\forall \mathrm{P}$. Assume that neither $\phi(\forall \mathrm{P})$ nor the formulas in lines L apart from (c)P in line $\mathrm{i}$ contain any occurrence of the singular argument $\mathrm{c}$. 


\begin{tabular}{llll}
\hline $\mathrm{i}$ & (i) & $(\mathrm{c}) \mathrm{P}$ & Premise \\
$\mathrm{L}$ & (j) & $\phi(\mathrm{c} / \mathrm{PP})$ & \\
$\mathrm{L}-\mathrm{i}$ & (k) & $\phi(\forall \mathrm{P})$ & $\forall \mathrm{I} \mathrm{i}, \mathrm{j}$ \\
\hline
\end{tabular}

Where ' $\mathrm{L}-\mathrm{i}$ ' is the possibly empty list of numbers occurring in $\mathrm{L}$ apart from $\mathrm{i}$.

- (Universal Elimination, $\forall \mathrm{E})$ Let $\phi(\forall \mathrm{P})$ be a formula governed by $\forall \mathrm{P}$.

\begin{tabular}{|c|c|c|c|}
\hline $\mathrm{L}_{1}$ & (i) & $\phi(\forall \mathrm{P})$ & \\
\hline $\mathrm{L}_{2}$ & (j) & (c) $\mathrm{P}$ & \\
\hline $\mathrm{L}_{1}, \mathrm{~L}_{2}$ & (k) & $\phi(\mathrm{c} / \forall \mathrm{P})$ & $\forall E \mathrm{i}, \mathrm{j}$ \\
\hline
\end{tabular}

- (Particular Introduction, $\exists \mathrm{I})$ Let $\phi(\exists \mathrm{P})$ be a formula governed by $\exists \mathrm{P}$.

\begin{tabular}{llll}
\hline $\mathrm{L}_{1}$ & (i) & $\phi(\mathrm{c} / \exists \mathrm{P})$ & \\
$\mathrm{L}_{2}$ & (j) & (c)P & \\
$\mathrm{L}_{1}, \mathrm{~L}_{2}$ & (k) & $\phi(\exists \mathrm{P})$ & $\exists \mathrm{I} \mathrm{i}, \mathrm{j}$ \\
\hline
\end{tabular}

- (Instantial Import, Imp) Let q stand for either $\exists$ or $\forall$, and $\phi(\mathrm{qP})$ be governed by qP. Assume c does not occur in $\phi(\mathrm{qP}), \psi$ or any of the formulas $\mathrm{L}_{1}$, and in no formula $\mathrm{L}_{2}$ apart from $\mathrm{j}$ and $\mathrm{k}$.

\begin{tabular}{llll}
\hline $\mathrm{L}_{1}$ & (i) & $\phi(\mathrm{qP})$ & \\
$\mathrm{j}$ & $(\mathrm{j})$ & $(\mathrm{c}) \mathrm{P}$ & Premise \\
$\mathrm{k}$ & $(\mathrm{k})$ & $\phi(\mathrm{c} / \mathrm{qP})$ & Premise \\
$\mathrm{L}_{2}$ & $(\mathrm{l})$ & $\psi$ & \\
$\mathrm{L}_{1}, \mathrm{~L}_{2}-\mathrm{j}-\mathrm{k}$ & $(\mathrm{m})$ & $\psi$ & Imp i, j, $\mathrm{k}, 1$ \\
\hline
\end{tabular}

As examples, I provide three proofs, which between them demonstrate all the derivation rules, apart from the rules for identity, which are not specific to Quarc. First, $(\forall \mathrm{S}) \mathrm{P} \vdash(\exists \mathrm{P}) \mathrm{S}$ :

\begin{tabular}{llll}
\hline 1 & $(1)$ & $(\forall \mathrm{S}) \mathrm{P}$ & Premise \\
2 & $(2)$ & aS & Premise \\
3 & $(3)$ & aP & Premise \\
2,3 & $(4)$ & $(\exists \mathrm{P}) \mathrm{S}$ & $\exists \mathrm{I} 2,3$ \\
1 & $(5)$ & $(\exists \mathrm{P}) \mathrm{S}$ & Imp $1,2,3,4$ \\
\hline
\end{tabular}

Secondly, the Aristotelian Barbara, i.e., $(\forall \mathrm{S}) \mathrm{M},(\forall \mathrm{M}) \mathrm{P} \vdash(\forall \mathrm{S}) \mathrm{P}$ : 


\begin{tabular}{llll}
\hline 1 & $(1)$ & $(\forall \mathrm{S}) \mathrm{M}$ & Premise \\
2 & $(2)$ & $(\forall \mathrm{M}) \mathrm{P}$ & Premise \\
3 & $(3)$ & $\mathrm{aS}$ & Premise \\
1,3 & $(4)$ & $\mathrm{aM}$ & $\forall \mathrm{E} 1,3$ \\
$1,2,3$ & $\mathrm{aP}$ & $\forall \mathrm{E} 2,4$ \\
1,2 & $(5)$ & $(\forall \mathrm{S}) \mathrm{P}$ & $\forall \mathrm{I} 3,5$ \\
\hline
\end{tabular}

And lastly, another Aristotelian conversion: No $\mathrm{P}$ is $\mathrm{S}$ follows from No $\mathrm{S}$ is $\mathrm{P}$. Instead of introducing into Quarc a negative quantifier translating 'no'-something that can be done- these sentences are translated here as synonymous with 'Every/any $\mathrm{S}$ is not $\mathrm{P}^{\prime},(\forall \mathrm{S}) \neg \mathrm{P}$ (this is similar to the way these sentences are formalised by the Predicate Calculus). We have to show that $(\forall \mathrm{S}) \neg \mathrm{P} \vdash(\forall \mathrm{P}) \neg \mathrm{S}$ :

\begin{tabular}{llll}
\hline 1 & $(1)$ & $(\forall \mathrm{S}) \neg \mathrm{P}$ & Premise \\
2 & $(2)$ & $\mathrm{aP}$ & Premise \\
3 & $(3)$ & $\mathrm{aS}$ & Premise \\
1,3 & $(4)$ & $\mathrm{a} \neg \mathrm{P}$ & $\forall \mathrm{E} 1,3$ \\
1,3 & $\neg \mathrm{aP}$ & $\mathrm{PS} 4$ \\
1,2 & $(5)$ & $\neg \mathrm{aS}$ & $\mathrm{PCR}$ ( $\neg$ Introduction) $3,2,5$ \\
1,2 & $(6)$ & $\mathrm{a} \neg \mathrm{S}$ & $\mathrm{SP} 6$ \\
1 & $(7)$ & $(\forall \mathrm{P}) \neg \mathrm{S}$ & $\forall \mathrm{I} \mathrm{2,7}$ \\
\hline
\end{tabular}

For additional examples, see (Ben-Yami 2014) and (Ben-Yami and Pavlovic, unpublished).

\subsection{Quarc and the predicate calculus}

Quarc was developed to model quantification in Natural Language, as well as other features of Natural Language, partly motivated by the feeling that the model the Predicate Calculus provides can be improved upon in important respects. A different question is, how does Quarc compare as a formal system with the Predicate Calculus, and whether it can shed any light on quantification as incorporated in the latter.

These questions were addressed primarily in Lanzet and Ben-Yami (2004) and Lanzet (2017). The latter paper uses anaphora, described above, and defining clauses, introduced there, to provide a translation of the first-order Predicate Calculus into its version of Quarc. It shows that its translation preserves truth-values and entailment, and it concludes that 'first-order predicate calculus and its devices of quantification can be seen as resulting from Quarc on certain semantic and syntactic restrictions, akin to simplifying assumptions' (abstract). Since then, a similar result was established for Quarc without defining clauses in an unpublished work by Hongkai Yin. As I do not use anaphora in the formal system of this paper, the reader is referred to Lanzet's and Yin's works for a full treatment of the comparative questions. What I shall do here is provide the principles of this comparison. These will suffice for the main purpose of this paper, analysing various modal issues from Quarc's point of view. 
The translations found in Lanzet and Ben-Yami (2004), using a precursor of Quarc, in Lanzet (2017) and in Yin (unpublished), although related, are different from each other, as are the languages used in these works. The translation I shall outline here is again related to all these versions yet not identical with any. All translations, however, are based on a similar analysis of the nature of quantification in the Predicate Calculus.

Let us examine the two Predicate Calculus formulas,

49. $\exists \mathrm{xP}(\mathrm{x})$

50. $\forall \mathrm{xP}(\mathrm{x})$

These can be read, 'Some things are P' and 'Everything is P', or 'Every-thing is P'. This every/some-thing reading can be adapted to any Predicate Calculus quantified sentence. Namely, the Predicate Calculus can be seen as allowing quantification only with one dedicated predicate, 'thing'. This predicate has the special property that, so to say, everything in the domain is a thing. We shall therefore add to Quarc a special unary predicate, 'Thing', abbreviated T, and aim to capture with it this property. Formally, this can be done in several ways. In this paper it is done by having the extension of $\mathrm{T}$ contain the extensions of all unary predicates and all singular arguments; and by 'c $\mathrm{T}$ ' being, for any singular argument, a theorem.

First, we add a derivation rule:

- (Thing Introduction, ThI) In any line of a proof, a formula of the form cT can be written, c being any SA, depending on no premise and with its justification being ThI.

\begin{tabular}{llll}
\hline- & (i) & cT & ThI \\
\hline
\end{tabular}

Secondly, extension of (Interpretation):

- $\mathrm{T}^{M}=\left\{\mathrm{c}^{M}: \mathrm{c}\right.$ is an SA of $\left.L\right\} \cup\left\{\alpha: \alpha \in \mathrm{P}^{M}\right.$ for some unary predicate $\mathrm{P}$ of $\left.L\right\}$

The idea behind the extension of (Interpretation) to $\mathrm{T}$ is that any named object is a Thing; and if an object is in the extension of any unary predicate $\mathrm{P}$-if it is a $\mathrm{P}$ - then it is also a Thing.

With Thing introduced into Quarc, we can translate formulas (49) and (50) as,

51. ( $\exists \mathrm{T}) \mathrm{P}$

52. $(\forall \mathrm{T}) \mathrm{P}$

The translation of more complex Predicate Calculus formulas will as a rule require the use of anaphors.

The Quarc predicate 'Thing' is not meant to capture the use of 'thing' in English, or of any other term I know in any natural language. Rather, it is meant to approximate the conception of quantification and the role of a domain in the standard semantics of the Predicate Calculus. In English, the term 'thing' can stand in for any classificatory term which one need not, cannot, or does not wish to specify, as in 'There are lots of things I'd like to buy'. ${ }^{3}$ The term 'thing' can also be a proxy for terms for actions,

\footnotetext{
${ }_{3}$ Definitions and examples in this paragraph are derived from Oxford Dictionaries Online, https://en. oxforddictionaries.com/definition/thing.
} 
events, thoughts or utterances, as in 'She said the first thing that came into her head' or 'The only thing I could do well was cook'; as well as terms for abstract entities or concepts, as in 'Mourning and depression are not the same thing'. And a philosopher may well open a metaphysics class by stating, 'Today we are going to discuss three things: non-being, empty space, and the relation between them'. Namely, rather than expressing a concept under which all particulars fall, 'thing' in English has no content that limits its application and therefore it can replace any specific term, much like the way 'it' or 'this' can be used as an anaphor for almost any word.

The fact that 'thing' has no specific content makes it appropriate for translating into English quantified Predicate Calculus formulas, in which what is quantified over is not specified by the formula. For this reason, we use the predicate 'Thing' in Quarc, which is meant to approximate the role of a domain in the Predicate Calculus (other approximations are also possible). It is relevant to note that the use of 'thing' in English is not matched by quantification in the Predicate Calculus, for it cautions us from assuming that with this use of ' $T$ ' we represent in Quarc a form of Natural Language quantification. 'Thing' is not needed for that purpose.

Quantification in the Predicate Calculus can now be seen as restricted in the following sense: Unlike Natural Language or Quarc, the Predicate Calculus allows quantification only with one concept, whose range contains at least all items denoted by any singular constant and all items belonging to any $n$-tuple in the range of any $n$-ary predicate. (This is not exactly the way the range of Quarc's T was defined, as the only predicates mentioned there were unary.) Put in these terms, it is hard to see any logical justification for such a restriction; surely it has nothing to do with the nature of quantification. It might be useful for some purposes, but it cannot be considered as capturing anything essential to quantification.

Nor should we think that the Predicate Calculus' way of incorporating quantification is the way to achieve absolute generality, a topic that has recently attracted some attention (Rayo and Uzquiano 2006). On the one hand, the domain of a Predicate Calculus model, which Quarc's Thing approximates, need not include absolutely everything: all it must include are the particulars in the range of constants and predicates (more accurate definition above), and these can be only few of the things one can otherwise talk about. Accordingly, this kind of inclusive domain is no guarantee for absolute generality. On the other hand, if talking about absolutely everything makes sense (we need not decide it here), a predicate with such a designation can be introduced into Quarc, by means of Thing or any other predicate letter, and absolute generality be captured by its means. So, the Predicate Calculus' way of incorporating quantification is not necessary for this end either.

We shall later use this analysis of quantification in the Predicate Calculus and its approximation by Quarc to analyse the modal issues with which the paper opened. For that purpose, we now proceed to introduce Modal Quarc.

\section{Modal Quarc}

Work on Modal Quarc is not as advanced as that on the non-modal versions introduced or mentioned above. Primarily, the completeness of the various systems hasn't yet been 
fully researched; it is not assumed below. Soundness, however, is simple to prove, and it suffices for our purposes here.

If we consider the use of modality in Natural Language, we shall see that, like negation, it has both a sentential use and a predicative one:

53. Necessarily, Socrates is human

54. Socrates is necessarily human

As with negation, the Predicate Calculus allows only the former, sentential use. Quarc, by contrast, again allows the unary sentential operator to function as a mode of predication as well:

55. $\square(\mathrm{sH})$

56. $\mathrm{s} \square \mathrm{H}$

As in the case of negation, if all arguments are singular, the sentential and predicative forms will be defined as equivalent, in both semantics and proof system. This equivalence, as we shall see, does not extend to the quantified case. Examples of the latter are:

57. Necessarily, all philosophers are mortal

58. $\square(\forall \mathrm{PM})$

59. All philosophers are necessarily mortal

60. $\forall \mathrm{P} \square \mathrm{M}$

The sentential versus predicative uses of the modal operators are Quarc's way of incorporating the de dicto-de re distinction. As seen from (55) and (56), the distinction exists, syntactically, also when all arguments are singular. And as Quarc's syntax suggests, if we are to remain closer to the syntax of Natural Language and moreover avoid apparent metaphysical implications, we better use not the phrase 'de re' or 'of the thing', but 'de predicatione' or 'of the predication', as did Avicenna (Movahed 2003), probably the first to note the distinction. ${ }^{4}$

Once several modes of predication are introduced - in our case, negation, possibility and necessitation - the option of their concatenation arises. As in Natural Language, this will be allowed in Quarc. For instance:

61. Socrates isn't possibly a dog

62. $s \neg \diamond \mathrm{D}$

63. Socrates possibly isn't Greek

64. $s \diamond \neg \mathrm{G}$

65. Some people are necessarily not Greek

66. $\exists \mathrm{P} \square \neg \mathrm{G}$

The length of such chains of operators is unbounded by the system's rules. Longer ones, as in the case of concatenation of sentential operators, may seem meaningless or at least hard to comprehend. For instance, we can now have not only $\diamond \diamond \neg \square \neg \neg \diamond(\mathrm{aP})$, but also, a $\diamond \diamond \neg \square \neg \neg \diamond$ P. Rules for the collapse and reordering of such concatenations can be proved and will depend on the specific modal system used. We shall not pursue this here.

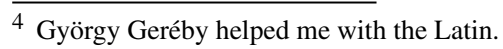




\subsection{Vocabulary and formula rules}

The language $L$ of Modal Quarc is thus enriched with two operators, $\square$ and $\diamond$. The unary sentential operators, namely $\neg, \square$ and $\diamond$, will also be called, predication operators.

As for formula formation rules, we shall replace the rule (negative predication) with a (modes of predication) rule, and add to (sentential operators):

- (Modes of predication) If $\mathrm{P}$ is an $n$-ary predicate, $\mathrm{c}_{1}, \ldots, \mathrm{c}_{\mathrm{n}} \mathrm{SAs}$, and * a chain of predication operators, then $\left(\mathrm{c}_{1}, \ldots, \mathrm{c}_{\mathrm{n}}\right) * \mathrm{P}$ is a formula.

- (Sentential operators) If $\phi$ is a formula, so are $\square(\phi)$ and $\nabla(\phi)$. The parentheses in both schemas are sentential parentheses.

Given the definition of governance, it follows that ' $\forall \mathrm{S}$ ' governs the formulas $(\forall S) \square P$ and $(\forall \mathrm{S}) \neg \square \neg \mathrm{P}$, for instance, but not the formulas $\square((\forall \mathrm{S}) \mathrm{P})$ or $\neg((\forall \mathrm{S}) \square \mathrm{P})$. In addition, in all these formulas the parentheses can be omitted without ambiguity: $\forall \mathrm{S} \square \mathrm{P}$, $\forall \mathrm{S} \neg \square \neg \mathrm{P}, \square \forall \mathrm{SP}, \neg \forall \mathrm{S} \square \mathrm{P}$.

\subsection{Semantics}

Extending the semantics to Modal Quarc, we add a non-empty set W of indices for different possible worlds or possibilities, and relativize truth to a possibility, w. We assume that a binary accessibility relation, $\mathbf{R}$, holds between elements of W. $<\mathrm{W}, \mathbf{R}>$ is called a frame. As usual, different constraints on $\mathbf{R}$ yield different modal systems.

(Interpretation) An interpretation for $L$ relative to a frame $<\mathrm{W}, \mathbf{R}>$ is a function $M$ for which the following conditions hold:

- The domain of $M$ is the set of all singular arguments and predicates of $L$, apart from $=$.

- For every $n$-ary predicate $\mathrm{R}$ in $L, \mathrm{R}^{M}$ is a set of $n+1$-tuples, $<\alpha_{1}, \ldots \alpha_{\mathrm{n}}$, w $>$, where $\mathrm{w} \in \mathrm{W}$.

- For any unary predicate $\mathrm{P}$ in $L$ and for any $\mathrm{w} \in \mathrm{W}$, there is some $\alpha$ such that $<\alpha, \mathrm{w}>\in \mathrm{P}^{M}$.

An interpretation does not relativize the value of singular arguments to possibilities, and in this sense, they are rigid. Also, since we use no domains of quantification, the question whether to use fixed or variable domains does not arise.

In addition, as above, the third, non-emptiness point is introduced, despite its artificiality, to simplify the semantics for the purposes of this paper. If it is not imposed, the most natural option is, I think, a three-valued semantics, which we shall not explore in this paper. Since below I primarily aim to show the invalidity of certain formulas, had we considered a three-valued semantics, which allows for additional truth-values of formulas (as truth-value in an interpretation is defined in the same way in it for the non-empty case), formulas that can be false and are therefore invalid on a two-valued semantics would remain invalid on it. The invalidity results established below are thus stronger than ones established for a three-valued semantics. Another question we shall not discuss, and for the same reason, is how to interpret the definitions below in case 
for no $\mathrm{v} \in \mathrm{W}, \mathrm{w} \mathbf{R} v$. We do not consider such models below; the consequences of relaxing this constraint haven't been fully explored.

For our purposes here, we extend (Interpretation) to the special predicate $\mathrm{T}$ or 'Thing':

- $\mathrm{T}^{M}=\left\{<\mathrm{c}^{M}, \mathrm{w}\right\rangle: \mathrm{c}$ is an SA of $L$ and $\left.\mathrm{w} \in \mathrm{W}\right\} \cup\left\{\langle\alpha, \mathrm{w}\rangle:\langle\alpha, \mathrm{v}\rangle \in \mathrm{P}^{M}\right.$ for some unary predicate $\mathrm{P}$ of $L$ and some $\mathrm{v} \in \mathrm{W}$, and $\mathrm{w} \in \mathrm{W}\}$

Namely, any named object is a Thing, in all possibilities; and if an object is in the extension of any unary predicate $\mathrm{P}$ in any possibility, then it is a Thing in all possibilities, although not in all of them it need be in P's extension. This interpretation rule is meant to reflect the option of a constant domain across possibilities in Modal Predicate Calculus, this choice made because the Barcan formulas and their converses are valid for such domains (see e.g. (Fitting 1999)). Other options can also be explored, but they will not be pursued here. Again, 'Thing' is introduced only to analyse quantification and modality in the Predicate Calculus; Quarc has no internal need for it.

(Truth-value in an interpretation) We define inductively the truth-value $[\phi, \mathrm{w}]^{M}$, or the truth-value of $\phi$ at $\mathrm{w}$ according to $M$, for any formula $\phi$ and any possibility w on an interpretation $M$. The truth-value $[\phi, \mathrm{w}]^{M}$ will always be either $\mathbf{T}$ or $\mathbf{F}$.

- (Basic formulas)

$\left[\left(\mathrm{c}_{1}, \ldots, \mathrm{c}_{n}\right) \mathrm{P}, \mathrm{w}\right]^{M}=\mathbf{T}$ if $<\mathrm{c}_{1}^{M}, \ldots, \mathrm{c}_{n}^{M}, \mathrm{w}>\in \mathrm{P}^{M}, \mathbf{F}$ otherwise.

$\left[\mathrm{c}_{1}=\mathrm{c}_{2}, \mathrm{w}\right]^{M}=\mathbf{T}$ if $\mathrm{c}_{1}^{M}$ is $\mathrm{c}_{2}^{M}, \mathbf{F}$ otherwise.

$\mathrm{c}_{i}$ here are any SAs, $n \geq 1$, and $\mathrm{P}$ any $n$-ary predicate.

- (Sentential operators)

$[\neg(\phi), \mathrm{w}]^{M}=\mathbf{T}$ if $[\phi, \mathrm{w}]^{M}=\mathbf{F}, \mathbf{F}$ otherwise.

$[(\phi) \rightarrow(\psi), \mathrm{w}]^{M}=\mathbf{F}$ if $[\phi, \mathrm{w}]^{M}=\mathbf{T}$ and $[\psi, \mathrm{w}]^{M}=\mathbf{F}, \mathbf{T}$ otherwise. Etc.

$[\square(\phi), \mathrm{w}]^{M}=\mathbf{T}$ if for every $\mathrm{v} \in \mathrm{W}$ for which $\mathrm{w} \mathbf{R} \mathrm{v},[\phi, \mathrm{v}]^{M}=\mathbf{T}, \mathbf{F}$ otherwise.

$[\diamond(\phi), \mathrm{w}]^{M}=\mathbf{T}$ if for some $\mathrm{v} \in \mathrm{W}$ for which $\mathrm{w} \mathbf{R} \mathrm{v},[\phi, \mathrm{v}]^{M}=\mathbf{T}, \mathbf{F}$ otherwise.

- (Modes of predication) Let \# be a predication operator, * a possibly empty chain of predication operators.

$$
\left[\left(\mathrm{c}_{1}, \ldots, \mathrm{c}_{n}\right) \# * \mathrm{P}, \mathrm{w}\right]^{M}=\left[\#\left(\mathrm{c}_{1}, \ldots, \mathrm{c}_{n}\right) * \mathrm{P}, \mathrm{w}\right]^{M}
$$

- (Quantification) If $\mathrm{P}$ is any unary predicate, $\mathrm{q}$ a quantifier, $\phi(\mathrm{qP})$ a formula governed by $\mathrm{qP}, \phi(\mathrm{x})$ is $\phi(\mathrm{x} / \mathrm{qP})$, and $\mathrm{w} \in \mathrm{W}$, then:

$[\phi(\forall \mathrm{P}), \mathrm{w}]^{M}=\mathbf{T}$ if $[\phi(\mathrm{x}), \mathrm{w}]$ is true in $M$ of every $\alpha$ such that $<\alpha, \mathrm{w}>\in \mathrm{P}^{M}$, F otherwise.

$[\phi(\exists \mathrm{P}), \mathrm{w}]^{M}=\mathbf{T}$ if $[\phi(\mathrm{x}), \mathrm{w}]$ is true in $M$ of some $\alpha$ such that $<\alpha, \mathrm{w}>\in \mathrm{P}^{M}$, F otherwise.

The definition of (true of/false of) should also be modified:

- (True of/false of) If $\phi(\mathrm{c})$ is a formula and $\phi(\mathrm{x})$ is the expression $\phi(\mathrm{x} / \mathrm{c})$, in which $\mathrm{x}$ replaced all occurrences of $\mathrm{c}$, then $[\phi(\mathrm{x}), \mathrm{w}]$ is true (false) of $\alpha$ in $M$, or $\phi(\mathrm{x})$ is true (false) in $M$ of $\alpha$ at w, if $[\phi(c), w]$ is true (false) in $M_{\alpha \mid c}$.

As can be seen from (modes of predication), Quarc's semantics offers a uniform analysis of the relation between the use of an operator as sentential and its use as mode of predication. In this respect, there is no distinction between $\neg, \square$ and $\diamond$. The 
former rule, (negative predication), is now seen as a specific case of the (modes of predication) one.

The definition of entailment should also be adjusted:

(Entailment) A set $\Gamma$ of formulas entails a formula $\phi$ relative to a frame $\mathrm{F}=$ $<\mathrm{W}, \mathbf{R}>$, or $\Gamma \vDash_{\mathrm{F}} \phi$, iff for every interpretation $M$ for $L$ relative to $\mathrm{F}$, if for any $\mathrm{W} \in \mathrm{W}$, for every $\psi \in \Gamma,[\psi, \mathrm{w}]^{M}=\mathbf{T}$, then $[\phi, \mathrm{w}]^{M}=\mathbf{T}$. A set $\Gamma$ of formulas entails a formula $\phi$ relative to a set of frames SF, or $\Gamma \vDash_{\text {SF }} \phi$, iff $\Gamma \vDash_{F} \phi$ for every $\mathrm{F}$ in SF.

\subsection{Proof system}

The derivation rules of Quarc that were introduced in the previous section are used in Modal Quarc as well. However, similarly to the semantics case, in which the rule (negative predication) was generalised to (modes of predication), the two derivation rules, (Sentence negation to Predication negation, SP) and (Predication negation to Sentence negation, PS), shall be generalised as well. The former rules shall be considered as specific cases of the rules, (Sentential operator to Predication operator, SP) and (Predication operator to Sentential operator, PS):

- (Sentential operator to Predication operator, SP) Let P be an $n$-ary predicate, $\mathrm{c}_{1}, \ldots$, $\mathrm{c}_{n} \mathrm{SAs}$, \# a predication operator, * a possibly empty chain of predication operators.

\begin{tabular}{llll}
\hline $\mathrm{L}$ & (i) & $\#\left(\mathrm{c}_{1}, \ldots, \mathrm{c}_{n}\right) * \mathrm{P}$ & \\
$\mathrm{L}$ & $(\mathrm{j})$ & $\left(\mathrm{c}_{1}, \ldots, \mathrm{c}_{n}\right) \# * \mathrm{P}$ & $\mathrm{SP} \mathrm{i}$ \\
\hline
\end{tabular}

- (Predication operator to Sentential operator, PS) Let P be an $n$-ary predicate, $\mathrm{c}_{1}, \ldots$, $\mathrm{c}_{n} \mathrm{SAs}$, \# a predication operator, * a possibly empty chain of predication operators.

\begin{tabular}{llll}
\hline $\mathrm{L}$ & (i) & $\left(\mathrm{c}_{1}, \ldots, \mathrm{c}_{n}\right) \# * \mathrm{P}$ & \\
$\mathrm{L}$ & (j) & $\#\left(\mathrm{c}_{1}, \ldots, \mathrm{c}_{n}\right) * \mathrm{P}$ & $\mathrm{PS}$ i \\
\hline
\end{tabular}

The derivation rules of Modal Propositional Logic are used in Modal Quarc as well. I give here three examples. A normal modal system is one that includes, in addition to the standard Propositional Calculus rules, the two rules Necessitation and K:

- (Necessitation, Nec) Let $\phi$ be a formula.

\begin{tabular}{llll}
\hline- & (i) & $\phi$ & \\
- & (j) & $\square(\phi)$ & Nec i \\
\hline
\end{tabular}

- (K) Let $\phi$ and $\psi$ be formulas.

\begin{tabular}{llll}
\hline $\mathrm{L}_{1}$ & (i) & $\square((\phi) \rightarrow(\psi))$ & \\
$\mathrm{L}_{2}$ & (j) & $\square(\phi)$ & \\
$\mathrm{L}_{1}, \mathrm{~L}_{2}$ & (k) & $\square(\psi)$ & $\mathrm{K} \mathrm{i}, \mathrm{j}$ \\
\hline
\end{tabular}


Additional rules are introduced according to the system in which we are interested, such as T, B, S4 or S5. The (T) rule, for instance, will be:

- (T) Let $\phi$ be a formula.

\begin{tabular}{llll}
\hline $\mathrm{L}$ & (i) & $\square(\phi)$ & \\
$\mathrm{L}$ & (j) & $\phi$ & $\mathrm{T}$ i \\
\hline
\end{tabular}

As $\diamond$ is never used in any proof below, we won't introduce any rules involving it to the proof system. It can be used as an abbreviation of $\neg \square \neg$. I nevertheless introduced it above to the vocabulary, formula rules and semantics, as it is used in some formalisations below and to show how Quarc handles it semantically.

\section{The Barcan formulas and their converses}

The Barcan formulas and their converses in Modal Predicate Calculus licence swapping the scope of quantifier and modal operator in some formulas. For instance, the Barcan formula (2) and its converse (4) license swapping the scope of universal quantifier and necessity operator between

67. $\forall x \square \phi(x)$

and

68. $\square \forall x \phi(x)$

Accordingly, the very idea of the Barcan formulas and their converses depends on specific syntactic features of the formal language under consideration, namely the Predicate Calculus: it results from the Predicate Calculus' incorporation of both quantification and modality by means of sentential operators.

When we turn to consider whether there are in Modal Quarc any formulas similar in significant respects to (67) and (68), we are faced with the difficulty that quantifiers are not sentential operators. Quantifiers occur in the argument position, while modal operators are used either as sentential or predication operators. Consequently, quantifier and modal operator cannot be syntactically swapped. What should count as Quarc formulas which relevantly resemble these Predicate Calculus formulas is therefore also a matter of interpretation.

We can take the Natural Language reading of formulas (67) and (68) as our guide. This makes sense also because an important object of our logical investigations is to study the logic of Natural Language, and to do that by formal means. With this in mind, we can read (67) and (68) as saying, 'Everything is necessarily $\phi$ ' and 'Necessarily everything is $\phi$ ', similarly to our readings of Predicate Calculus formulas above. We can now proceed in two ways.

One way, which we shall pursue later, is to translate these formulas into Quarc by means of the predicate 'Thing'. This, however, although it may provide us with an analysis, from Quarc's point of view, of the Predicate Calculus formulas, will not yield formulas similar in their standing in Quarc to that of (67) and (68) in the Predicate 
Calculus: 'Thing' was introduced to Quarc not because of any internal need of the system but only in order to reflect quantification as incorporated in the Predicate Calculus. And we have also argued that this use of 'Thing' in quantification does not reflect the use of 'thing' or any other term in English.

To find Quarc formulas that relate to each other syntactically in a way similar to that in which (67) and (68) do, and which use the apparatus integral to Quarc, we may take as our lead the observation that 'thing' is in fact used, in English, to stand in for any classificatory term which one need not, cannot, or does not wish to specify (see above). Accordingly, we may consider 'Every-thing is necessarily...' and 'Necessarily every-thing is...' as standing in for 'Every $\mathrm{S}$ is necessarily...' and 'Necessarily every $\mathrm{S}$ is...' in English (and with appropriate changes in other natural languages), where ' $\mathrm{S}$ ' is any unary predicate. This suggests the sentences,

69. Every $\mathrm{S}$ is necessarily $\mathrm{P}$

70. Necessarily, every $\mathrm{S}$ is $\mathrm{P}$

Formalised by,

71. $\forall \mathrm{S} \square \mathrm{P}$

72. $\square \forall \mathrm{SP}$

These sentences use unary predicates. More complex sentences of similar forms will be, for instance, 'Every S necessarily R's some P' and its translation, $(\forall S, \exists P) \square R$; and 'Necessarily, every S R's some P' and $\square(\forall S, \exists P) R$. For our purposes here it will suffice to investigate the simplest case.

Formulas (71) and (72) of Quarc relate to each other, syntactically, in a way resembling that in which formulas (67) and (68) do, in the following respect as well. (71) is generated from ' $\mathrm{a} \square \mathrm{P}$ ' through (Quantification) by introducing the quantified argument ' $\forall \mathrm{S}$ ', and ' $\mathrm{a} \square \mathrm{P}$ ' is generated from ' $\mathrm{aP}$ ' by (Modes of predication). Namely, in the process of generation of (71), quantification is the last operator to be introduced, preceded by the introduction of the necessity operator; with appropriate changes, this is the case of (67) as well. We can see the relation of operator scope as a specific case of this relation of order of their introduction, with 'last introduced' generalising 'widest scope'. It is the other way around with both (72) and (68).

The Quarc formulas we shall consider as resembling in their syntactical structure the Barcan formulas and their converses are therefore,

73. $\forall \mathrm{S} \square \mathrm{P} \rightarrow \square \forall \mathrm{SP}$

74. $\square \forall \mathrm{SP} \rightarrow \forall \mathrm{S} \square \mathrm{P}$

I shall show that these are both unprovable and invalid in Quarc. Similar considerations apply to their corresponding particular versions:

75. $\diamond \exists \mathrm{SP} \rightarrow \exists \mathrm{S} \diamond \mathrm{P}$

76. $\exists \mathrm{S} \diamond \mathrm{P} \rightarrow \diamond \exists \mathrm{SP}$

As the same considerations apply to both pairs (a fact I won't show here), it is enough to consider the former one.

It is straightforward to show that Modal Quarc is sound, and therefore showing that these formulas are invalid will also establish that they are unprovable. Still, it 
is informative to see why Quarc has no proofs analogous to those of the Predicate Calculus. I start by examining this.

The proof of the converse Barcan formula (4), $\square \forall \mathrm{x} \phi(\mathrm{x}) \rightarrow \forall \mathrm{x} \square \phi(\mathrm{x})$, is simple. Here it is, with a proof system for Modal Predicate Calculus adapting the one introduced above for Modal Quarc:

\begin{tabular}{llll}
\hline 1 & $(1)$ & $\square \forall \mathrm{x} \phi \mathrm{x}$ & Premise \\
2 & $(2)$ & $\forall \mathrm{x} \phi \mathrm{x}$ & Premise \\
2 & $(3)$ & $\phi \mathrm{a}$ & $\forall \mathrm{E} 2$ \\
& $(4)$ & $\forall \mathrm{x} \phi \mathrm{x} \rightarrow \phi \mathrm{a}$ & $\rightarrow \mathrm{I} 2,3$ \\
& $(5)$ & $\square(\forall \mathrm{x} \phi \mathrm{x} \rightarrow \phi \mathrm{a})$ & $\mathrm{Nec} 4$ \\
1 & $(6)$ & $\forall \phi \mathrm{a}$ & $\mathrm{K} 1,5$ \\
1 & $(7)$ & $\square \forall \square \phi \mathrm{x}$ & $\forall \mathrm{I} 6$ \\
& $(8)$ & & $\rightarrow \mathrm{I} 1,7$ \\
\hline
\end{tabular}

The only modal derivation rules used are Nec and K. Let us now try to adapt this proof to Quarc. We examine whether $\vdash \square \forall \mathrm{SP} \rightarrow \forall \mathrm{S} \square \mathrm{P}$ by a proof which follows the one above.

\begin{tabular}{llll}
\hline 1 & $(1)$ & $\square \forall \mathrm{SP}$ & Premise \\
2 & $(2)$ & $\forall \mathrm{SP}$ & Premise \\
3 & $(3)$ & $\mathrm{aS}$ & Premise \\
2,3 & $\mathrm{aP}$ & $\forall \mathrm{E}, 2,3$ \\
3 & $(4)$ & $\forall \mathrm{SP} \rightarrow \mathrm{aP}$ & $\rightarrow \mathrm{I} 2,4$ \\
\hline
\end{tabular}

But now, unlike at the parallel stage in the former proof, we cannot apply Nec to step (5) and obtain $\square(\forall \mathrm{SP} \rightarrow \mathrm{aP})$, because the formula in line (5) is not a theorem but depends on line (3).

This Predicate-Calculus-style proof was blocked because, in order to use the universal elimination or introduction rules in Quarc, we must assume that a specific predicate applies to a singular argument ('aS' in line (3)). And although the universal statement to be used is given as necessary, that application of the predicate to the argument is not. This of course does not show that no proof is possible; as said above, this follows from soundness together with the invalidity, to which I know turn.

I shall show that neither of the formulas $\forall \mathrm{S} \square \mathrm{P}$ and $\square \forall \mathrm{SP}$ entails the other, even in S5 (namely, the set of frames that consists of all frames in which the accessibility relation is reflexive, symmetric and transitive), the strongest modal system standardly considered. To do that, I provide two interpretations, each being a counterexample to one entailment. For both interpretations, the set of possibilities $\mathrm{W}$ consists of two possibilities, w1 and w2, each possibility accessible from both possibilities. The accessibility relation is thus reflexive, symmetric and transitive, and it therefore qualifies as an S5 frame. We consider only two singular arguments of the language, $a$ and $b$, and for both interpretations, $M$ and $N, \mathrm{a}^{M}=\mathrm{a}^{N}=\mathrm{a}$ and $\mathrm{b}^{M}=\mathrm{b}^{N}=\mathrm{b}$. We also consider only two, unary predicates, $\mathrm{S}$ and $\mathrm{P}$. 
First, let us show that $\nvdash_{\mathrm{S} 5} \forall \mathrm{S} \square \mathrm{P} \rightarrow \square \forall \mathrm{SP}$. Let the interpretation $M$ be defined as follows on the predicates $\mathrm{S}$ and $\mathrm{P}$ :

$$
\mathrm{S}^{M}=\{<\mathrm{a}, \mathrm{w} 1>,<\mathrm{b}, \mathrm{w} 2>\}, \mathrm{P}^{M}=\{<\mathrm{a}, \mathrm{w} 1>,<\mathrm{b}, \mathrm{w} 1>,<\mathrm{a}, \mathrm{w} 2>\}
$$

The interpretation is defined on both singular arguments and on both predicates; moreover, for $\mathrm{S}$, for both $\mathrm{w} 1$ and $\mathrm{w} 2$ there is some $\alpha$ such that $\left\langle\alpha, \mathrm{w} i>\in \mathrm{S}^{M}\right.$, and similarly for P. The interpretation thus fulfils the three conditions imposed on a Modal Quarc interpretations above. (In later proofs, we won't show that the interpretations fulfil these conditions.)

(1) $\forall \mathrm{S} \square \mathrm{P}$ is true at w1 in $M,[\forall \mathrm{S} \square \mathrm{P}, \mathrm{w} 1]^{M}=\mathbf{T}$, just in case $[\mathrm{x} \square \mathrm{P}, \mathrm{w} 1]$ is true in $M$ of every element $<\alpha, w 1>$ of $S^{M}$, which is only $<$ a, w1 $>$. According to (modes of predication), $\mathrm{a} \square \mathrm{P}$ is true at w just in case $\square \mathrm{aP}$ is. Since both w1 and w2 are all the possibilities accessible from $\mathrm{w} 1, \square \mathrm{aP}$ is true at $\mathrm{w} 1$ iff aP is true at $\mathrm{w} 1$ and w2. But this is so. So $\forall \mathrm{S} \square \mathrm{P}$ is true at w1.

(2) $\square \forall \mathrm{SP}$ is true at w1 just in case $\forall \mathrm{SP}$ is true at $\mathrm{w} 1$ and at w2. $\forall \mathrm{SP}$ is true at w2 just in case $\mathrm{xP}$ is true of every element $<\alpha$, w $2>$ of $\mathrm{S}^{M}$, which is only $<\mathrm{b}, \mathrm{w} 2>$. But $\mathrm{xP}$ is false of $\mathrm{b}$ at $\mathrm{w} 2$. So $\square \forall \mathrm{SP}$ is false at $\mathrm{w} 1$.

(3) Accordingly, $\forall \mathrm{S} \square \mathrm{P} \rightarrow \square \forall \mathrm{SP}$ is false at w1 in $M$, and $\nvdash_{\mathrm{S} 5} \forall \mathrm{S} \square \mathrm{P} \rightarrow \square \forall \mathrm{SP}$.

Secondly, we show that $\nvdash_{\mathrm{S} 5} \square \forall \mathrm{SP} \rightarrow \forall \mathrm{S} \square \mathrm{P}$. Let the interpretation $N$ be defined as follows on $\mathrm{S}$ and $\mathrm{P}$ :

$\mathrm{S}^{N}=\{<\mathrm{a}, \mathrm{w} 1>,<\mathrm{b}, \mathrm{w} 2>\}, \mathrm{P}^{N}=\{<\mathrm{a}, \mathrm{w} 1>,<\mathrm{b}, \mathrm{w} 1>,<\mathrm{b}, \mathrm{w} 2>\}$

(1) $\square \forall \mathrm{SP}$ is true at $\mathrm{w} 1$ in $N$ just in case $\forall \mathrm{SP}$ is true at w1 and at w2. $\forall \mathrm{SP}$ is true at $\mathrm{w} 1$ just in case $\mathrm{xP}$ is true of every element $<\alpha, \mathrm{w} 1>$ of $\mathrm{S}^{M}$, which is only $<\mathrm{a}, \mathrm{w} 1>$, and $\mathrm{xP}$ is indeed true of a at $\mathrm{w} 1 ; \forall \mathrm{SP}$ is true at $\mathrm{w} 2$ just in case $\mathrm{xP}$ is true of every element $<\alpha, \mathrm{w} 2>$ of $\mathrm{S}^{M}$, which is only $<\mathrm{b}, \mathrm{w} 2>$, and $\mathrm{xP}$ is indeed true of $\mathrm{b}$ at $\mathrm{w} 2$. So $\square \forall \mathrm{SP}$ is true at w1.

(2) $\forall \mathrm{S} \square \mathrm{P}$ is true at $\mathrm{w} 1$ just in case $\mathrm{x} \square \mathrm{P}$ is true at $\mathrm{w} 1$ of every element $<\alpha, \mathrm{w} 1>$ of $\mathrm{S}^{M}$, which is only $<\mathrm{a}, \mathrm{w} 1>$. According to (modes of predication), $\mathrm{a} \square \mathrm{P}$ is true at $\mathrm{w}$ just in case $\square \mathrm{aP}$ is. Since $\mathrm{w} 1$ and $\mathrm{w} 2$ are all the possibilities accessible from $w 1, \square \mathrm{aP}$ is true at $\mathrm{w} 1$ iff aP is true at $\mathrm{w} 1$ and $\mathrm{w} 2$. But aP is false at w2, so $\forall \mathrm{S} \square \mathrm{P}$ is false at $\mathrm{w} 1$.

(3) Accordingly, $\square \forall \mathrm{SP} \rightarrow \forall \mathrm{S} \square \mathrm{P}$ is false at w1 in $N$, and $\nvdash_{\mathrm{S} 5} \square \forall \mathrm{SP} \rightarrow \forall \mathrm{S} \square \mathrm{P}$.

Thus, the Quarc formulas (73) and (74), which resemble in their syntactical features the Barcan formulas and their converses, are invalid.

The way Modal Quarc invalidates its formulas (73) and (74) can be related to the way this can be achieved by Modal Predicate Calculus for the Barcan formulas and their converses. In Modal Predicate Calculus, this can be done by allowing varying domains of quantification between worlds. Quarc, by contrast, has no domain of quantification; instead, the plurality over which the sentences we considered quantify is determined as the extension of the unary predicate which is part of the quantified argument. Since this extension can vary between possibilities, this enables Modal Quarc to vary between possibilities the pluralities over which a given sentence quantifies. Moreover, this is done without the dilemma between allowing either variable or fixed 
domains ever arising: predicates naturally apply to different particulars at different possibilities. In the interpretations above, providing counterexamples to Quarc's formulas was achieved by having the extension of $\mathrm{S}$ being $\{<\mathrm{a}, \mathrm{w} 1\rangle,<\mathrm{b}, \mathrm{w} 2\rangle\}$, namely, different for different possibilities. In addition, since these variable pluralities need not contain the values of singular arguments, there is no need to disallow the latter, as is done on Kripke's approach to variable domains. ${ }^{5}$

The difference between the logical status of (1)-(4) in Modal Predicate Calculus and that of (73)-(76) in Modal Quarc is a result of their different conception of quantification. This can be seen if we try to approximate the formulas of the one calculus in the other. I start with an approximate translation of the Quarc formulas into the Predicate Calculus. Formulas (71) and (72) of Quarc, $\forall \mathrm{S} \square \mathrm{P}$ and $\square \forall \mathrm{SP}$, translate English's (69) and (70), 'Every S is necessarily P' and 'Necessarily, every S is P'. The standard translations of these sentences into the Predicate Calculus are,

77. $\forall \mathrm{x}(\mathrm{Sx} \rightarrow \square \mathrm{Px})$

78. $\square \forall \mathrm{x}(\mathrm{Sx} \rightarrow \mathrm{Px})$

These formulas are not equivalent to Quarc's (71) and (72): for instance, if there is no particular in the extension of $S$ at w1, then according to the three-valued semantics suggested above, the truth-value at w1 of (71), $\forall \mathrm{S} \square \mathrm{P}$, is undefined, while (77) is true. We cannot therefore adequately translate Quarc into the Predicate Calculus in this way. Still, for this approximation of the Quarc formulas, neither formula entails the other, as can be shown by adapting to the Predicate Calculus the interpretations we used above. To show this, we use the same frame $\mathrm{W}$ as above, consisting of two possibilities, w1 and w2, each accessible to both possibilities. Our interpretation $M$ is:

$$
\mathrm{S}^{M}=\{<\mathrm{a}, \mathrm{w} 1>,<\mathrm{b}, \mathrm{w} 2>\}, \mathrm{P}^{M}=\{<\mathrm{a}, \mathrm{w} 1>,<\mathrm{b}, \mathrm{w} 1>,<\mathrm{a}, \mathrm{w} 2>\}
$$

As can be easily checked, (77) is true at w1, while (78) is false. Accordingly,

$$
\nvdash_{\mathrm{S} 5} \forall \mathrm{x}(\mathrm{Sx} \rightarrow \square \mathrm{Px}) \rightarrow \square \forall \mathrm{x}(\mathrm{Sx} \rightarrow \mathrm{Px}) .
$$

A similar adaptation of $N$ above will show that

$$
\not_{\mathrm{S} 5} \square \forall \mathrm{x}(\mathrm{Sx} \rightarrow \mathrm{Px}) \rightarrow \forall \mathrm{x}(\mathrm{Sx} \rightarrow \square \mathrm{Px}) .
$$

Namely, if we represent in this way in the Predicate Calculus the restricted quantification of Quarc, then the translations of the Quarc's formulas (73)-(76) are also invalid. These approximations do not provide instances of the Barcan formulas or of their converses, as the string of symbols ( $\mathrm{Sx} \rightarrow \square \mathrm{Px}$ ) of (77) is not in the scope of $\square$.

Approximating the Predicate Calculus formulas in Quarc sheds additional light on the nature of quantification in the former and the reasons for the validity of the Barcan formulas and their converses in it, for constant domains. The approximation is achieved with the special unary predicate, 'Thing' or T, introduced above. As mentioned there, a full translation of the Predicate Calculus formulas uses anaphora, so here I shall only consider, as an illustration, the translation of the simple Predicate Calculus formulas,

79. $\forall \mathrm{x} \square \mathrm{P}(\mathrm{x})$

80. $\square \forall \mathrm{xP}(\mathrm{x})$

\footnotetext{
5 The formulation in this paragraph is indebted to discussions with Lanzet.
} 
These are read, 'Every-thing is necessarily P' and 'Necessarily, every-thing is P'. These English sentences are translated into Quarc as:

\section{1. $\forall \mathrm{T} \square \mathrm{P}$}

82. $\square \forall \mathrm{TP}$

And these formulas are indeed equivalent. For instance, the former can be proved from the latter already in $\mathrm{K}$, or equivalently, $\vdash_{\mathrm{K}} \square \forall \mathrm{TP} \rightarrow \forall \mathrm{T} \square \mathrm{P}$ :

\begin{tabular}{llll}
\hline 1 & $(1)$ & $\square \forall \mathrm{TP}$ & Premise \\
2 & $(2)$ & $\forall \mathrm{TP}$ & Premise \\
& $(3)$ & $\mathrm{aT}$ & $\mathrm{ThI}$ \\
2 & $(4)$ & $\mathrm{aP}$ & $\forall \mathrm{E}, 2,3$ \\
& $(5)$ & $\forall \mathrm{TP} \rightarrow \mathrm{aP}$ & $\rightarrow \mathrm{I} 2,4$ \\
& $(6)$ & $\square(\forall \mathrm{TP} \rightarrow \mathrm{aP})$ & $\mathrm{Nec} 5$ \\
1 & $(7)$ & $\square \mathrm{aP}$ & $\mathrm{K} 6,1$ \\
1 & $(8)$ & $\mathrm{a} \square \mathrm{P}$ & $\mathrm{SP} 7$ \\
9 & $(9)$ & $\mathrm{aT}$ & Premise \\
1 & $(10)$ & $\square \mathrm{T} \square \mathrm{P}$ & $\forall \mathrm{I} 9,8$ \\
& $(11)$ & $\square \forall \mathrm{TP} \rightarrow \forall \mathrm{T} \square \mathrm{P}$ & $\rightarrow \mathrm{I}, 1,10$ \\
\hline
\end{tabular}

The proof of $\forall \mathrm{T} \square \mathrm{P} \rightarrow \square \forall \mathrm{TP}$, which can be carried out in S5, will not be provided here, as it is much longer, as is the proof of the Barcan formula of the Predicate Calculus it translates (first found in Prior 1956; see also Menzel 2018).

The formulas' validity follows from the soundness of the proof system, which is easy to establish (as mentioned above, the completeness of the various modal systems hasn't been fully researched). But to demonstrate the semantics of T, we show directly that $\vDash_{\mathrm{K}} \forall \mathrm{T} \square \mathrm{P} \rightarrow \square \forall \mathrm{TP}$. For this entailment to hold in $\mathrm{K}$, we make no assumption on the accessibility relation $\mathbf{R}$.

Suppose that $[\forall \mathrm{T} \square \mathrm{P}, \mathrm{w}]$ is true in $M$; we have to show that so is [ $\square \forall \mathrm{TP}, \mathrm{w}]$. Namely, we should show that for any $\mathrm{v}$ accessible from $\mathrm{w},[\forall \mathrm{TP}, \mathrm{v}]$ is true in $M$. This is so iff, for any such $\mathrm{v},[\mathrm{xP}, \mathrm{v}]$ is true in $M$ of every element $<\alpha, \mathrm{v}>$ of $\mathrm{T}^{M}$. Namely, for every element $<\alpha, \mathrm{v}>$ of $\mathrm{T}^{M}$, [cP, v] should be true in $M_{\alpha \mid \mathrm{c}}$-an interpretation for which the value of $\mathrm{c}$ is $\alpha$ and which coincides with $M$ on any other input. That is, for every element $<\alpha, \mathrm{v}>$ of $\mathrm{T}^{M}$, it should be the case that $<\alpha, \mathrm{v}>\in \mathrm{P}^{M}$.

Since we assume that $[\forall \mathrm{T} \square \mathrm{P}, \mathrm{w}]$ is true in $M$, it similarly follows that $[\mathrm{c} \square \mathrm{P}, \mathrm{w}]$ is true in $M_{\alpha \mid \mathrm{c}}$ of every element $<\alpha, \mathrm{w}>$ of $\mathrm{T}^{M}$. But $[\mathrm{c} \square \mathrm{P}, \mathrm{w}]^{M}=[\square \mathrm{cP}, \mathrm{w}]^{M}$, so it follows that $[\square \mathrm{cP}, \mathrm{w}]$ is true in $M_{\alpha \mid c}$ of every element $<\alpha, \mathrm{w}>$ of $\mathrm{T}^{M}$. Namely, for any $\mathrm{v}$ accessible from $\mathrm{w},[\mathrm{cP}, \mathrm{v}]$ is true in $M_{\alpha \mid c}$ of every element $<\alpha, \mathrm{w}>$ of $\mathrm{T}^{M}$, or $<\alpha, \mathrm{v}>\in \mathrm{P}^{M}$.

Now, for any $M, \mathrm{~T}^{M}=\left\{\left\langle\mathrm{c}^{M}, \quad \mathrm{w}>\right.\right.$ : $\mathrm{c}$ is an SA of $L$ and $\mathrm{W} \in \mathrm{W}\} \cup\left\{\langle\alpha, \mathrm{w}\rangle:\langle\alpha, \mathrm{v}\rangle \in \mathrm{S}^{M}\right.$ for some $\mathrm{v} \in \mathrm{W}$, and $\left.\mathrm{w} \in \mathrm{W}\right\}$. Accordingly, the extension of $\mathrm{T}$ is not a function of $\mathrm{w}$; or, for any $\mathrm{w}$ and $\mathrm{v},<\alpha, \mathrm{w}>\in \mathrm{T}^{M}$ iff $<\alpha, \mathrm{v}>\in \mathrm{T}^{M}$. Accordingly, if $<\alpha, \mathrm{v}>\in \mathrm{T}^{M}$ for some $\mathrm{v}$ accessible from $\mathrm{w}$, it 
follows that $<\alpha, \mathrm{w}\rangle \in \mathrm{T}^{M}$. So, if $[\mathrm{cP}, \mathrm{v}]$ is true in any interpretation that coincides with $M$ on any input apart from possibly c, which it sends to an element $\alpha$ such that $\langle\alpha, \mathrm{w}\rangle \in \mathrm{T}^{M},[\mathrm{cP}, \mathrm{v}]$ is also true in any such interpretation that sends $\mathrm{c}$ to an element $\alpha$ such that $<\alpha, \mathrm{v}>\in \mathrm{T}^{M}$, or, for any such element, $<\alpha, \mathrm{v}>\in \mathrm{P}^{M}$. QED

It has been argued in Lanzet (2017) that the Predicate Calculus can be viewed as a restricted version of Quarc, resulting from it by limiting quantification to quantification over 'Things', namely only by means of the unary predicate T. The current paper captured this claim through the introduction of the unary predicate $\mathrm{T}$ with the special semantic property specified above in the extension of (Interpretation) to it. ${ }^{6}$ We have now arrived at a related conclusion: formulas of Quarc that resemble, syntactically, the Barcan formulas and their converses are invalid. Nor should the Barcan formulas or their converses be considered as representing the meaning of idiomatic Natural Language sentences (cf. the discussion of 'Thing' vs. the English 'thing'). The validity of these formulas in the Predicate Calculus results from limiting quantification in it to one done with a single, special predicate, whose domain is constant across possibilities and which applies to all named objects and all things that ever fall under any unary predicate. We should not consider this validity as reflecting any fact about quantification, modality or their interaction in Natural Language, and we should not consider it a result of an intuitive incorporation of modality or quantification in a formal language.

\section{Necessary existence}

'Ordinary modal logic', wrote Prior, 'is haunted by the myth that whatever exists exists necessarily' (1957, p. 48). Here is one way in which the spectre of necessary existence arises. To say that Socrates exists necessarily is to say, in 'ordinary modal logic', that necessarily, there is something which is Socrates, or that $\square \exists x(x=s)$; and this can be proved:

\begin{tabular}{lll}
\hline$(1)$ & $\mathrm{s}=\mathrm{s}$ & $=\mathrm{I}$ \\
$(2)$ & $\exists \mathrm{x}(\mathrm{x}=\mathrm{s})$ & $\exists \mathrm{I} 1$ \\
$(3)$ & $\square \exists \mathrm{x}(\mathrm{x}=\mathrm{s})$ & $\mathrm{Nec} 2$ \\
\hline
\end{tabular}

Introducing the universal quantifier and then applying necessitation again, one also proves that,

\section{3. $\square \forall \mathrm{y} \square \exists \mathrm{x}(\mathrm{x}=\mathrm{y})$,}

or that necessarily everything necessarily exists._Let us examine whether Quarc can help exorcise this apparition.

When we turn to Quarc, the very existence of a formula resembling (83) in both structure and what it says is doubtful. Quarc distinguishes in its formalisation three

\footnotetext{
6 The incorporation of this claim in (Lanzet 2017) is different in some respects, but I think this does not affect the conclusions here.
} 
different constructions in Natural Language which have commonly been merged in the tradition following Frege's Begriffsschrift. These are,

a. particular quantification

b. 'exist' as predicate

c. the 'there is' construction

The fact that in Natural Language these three constructions are syntactically different from each other is clear. Instances of them in English are,

84. Some Greeks are philosophers

85. Abraham existed, Adam didn't

86. There are giraffes, but no unicorns

Syntactically, 'some' is a quantifier, similar in grammatical role to other quantifier words and phrases like 'all', 'most', 'three', 'at least five', 'infinitely many' and others. It is a one-place determiner, combining with a noun to form a noun phrase. 'Exist', by contrast, is an unexceptional predicate. 'There is/are' is neither a quantifier nor a predicate. This seems to be a universal of Natural Language. ${ }^{7}$

The Aristotelian tradition did not relate particular quantification to either existence predication or the 'there is' construction, and it did not have an existential quantifier. Indeed, there is no reason to conceive of the existential import, if any, of Natural Language sentences that use the particular quantifier as different from that of sentences using the universal or any other quantifier. And I added, if any, since sentences like the following one are idiomatic:

\section{Some biblical characters never existed. For instance, Adam didn't.}

It therefore seems that quantification in Natural Language has no existential import. Accordingly, if our formal quantificational system aims at representing the logic of Natural Language, it shouldn't treat the particular quantifier as existential, it shouldn't have an existential quantifier, and none of its quantifiers should have existential import. $^{8}$

As mentioned, the use of 'exist' as a predicate - a verb in English and many other languages - is, grammatically, unexceptional. To be meaningful, it presupposes that we can refer to things that do not exist: (85) and (87), if true, refer to Adam and truly say that he did not exist. Although I don't find this reference problematic, some have. I won't

\footnotetext{
7 See (Peters \& Westerståhl 2006) for 'some', (McNally 2011) for 'there is' and 'exists'.

8 McGinn (2000, Chap. 2) has also argued that 'some' in Natural Language has no existential import and that 'exist' is a predicate. He does not distinguish, however, between the 'there is' construction and quantification. Moreover, he adopts the Predicate Calculus as his logic, reinterpreting it, while this paper considers an alternative system.

Graham Priest has similarly claimed that the particular quantifier has no existential import; see, for instance, (Priest 2016: Ch. 18), where the history of the opposite view is also considered in some detail. But like McGinn, he does this within the framework of the Predicate Calculus, whose quantifier he reinterprets. By contrast, I think that the Predicate Calculus also deviates from the way Natural Language incorporates quantification. Moreover, once the Predicate Calculus' $\exists$ has been identified with the particular quantifier, it is difficult to introduce an additional, non-quantificational construction, capturing 'there is' sentences and distinguished from both particular quantification and predication of existence. Indeed, Priest collapses the 'there is' construction into a different way of predicating existence. See (Priest 2016: passim, especially Chap. 17).
} 
digress to defend it in detail against all possible objections, but I offer a diagnosis of one line of thought that may have contributed to this view, as it relates to the alternative conceptual framework offered by Quarc. Existence, on the standard interpretation of the Predicate Calculus, is captured by the existential quantifier. Accordingly, given the standard model-theoretic semantics of this calculus, the items in the domain, over which this quantifier ranges and which verify formulas of the form $\exists x \phi x$, have been taken to be things that exist. Next, names, in that calculus, refer to items in the domain, and therefore reference has also been taken to be to things that exist. Consequently, reference to non-existent things appeared problematic. (Free logic (Lambert 2003, Nolt 2018) tried to introduce some distinctions here, but it has never become the standard or even a widespread way of thinking about these issues.) By contrast, if quantification has no existential import, as in Quarc and, arguably, in Natural Language, then what we quantify over-which may also be fictional characters, pagan gods or biblical characters - need not exist. Accordingly, reference, which may also be to such things, does not presuppose existence either.

The grammar of the constructions that translate the English 'there is/are' into different languages shows more variability than that of the translations of 'some' or 'exist'. The English expletive 'there', for instance, has no parallel in Hebrew or Hungarian; and German uses not the verb 'be' but 'give' (geben). ${ }^{9}$ In this paper, I shall not analyse its logic or incorporate it in Quarc. For my purposes here, it suffices to note that the existential import of this construction has been challenged, for instance by Azzouni (2007) and Crane (2012, 2013 Ch. 2). ${ }^{10}$ The lack thereof, at least for some uses of 'there is/are', can be demonstrated by sentences like,

88. There are some biblical characters who never existed, while there are many who did.

'There is' sentences are usually called in linguistics, existential sentences. However, since they are not attributions of existence, this term is inappropriate. We better call them, instantial sentences. ${ }^{11}$ This phrase also accords with the following use:

89. There were several important Roman philosophers; for instance, Lucretius and Cicero.

The distinction between an item's being an instance of a predicate and that item's existence - the former being possible without the latter, as is the case with 'Adam' in (87) — and Quarc's adoption of this distinction, makes Quarc have an Instantial Import derivation rule, not an existential one. Similarly, the non-emptiness rule imposed for simplification in the semantics should be seen as requiring that every unary predicate have instances, but not that these instances exist. For this reason, the rule was called 'Instantiation' in Ben-Yami (2014, p. 130).

\footnotetext{
9 For this variability and for current views in linguistics on the 'there is' construction, see (McNally 2011).

10 Neither shall I discuss in this paper the implications of the position developed here for Quine's view of the relation of ontology to quantification. His view is criticised in the works by Azzouni and Crane which were just mentioned, but my position is not identical to theirs. It would have forced us to digress to semantical considerations which are largely irrelevant to the central issues of this paper.

11 This terminology was already suggested by Strawson (1959: 241) and later adopted by McNally (1998). My intention is different from theirs in important respects, but I leave its elaboration for a different occasion.
} 
As mentioned, Natural Language distinguishes, syntactically, between particular quantification, instantial sentences, and predication using 'exist'; and we just saw that arguably, the first two are also independent in their meaning from ascription of existence. (The relation between particular quantification and instantial sentences will not be discussed here; see some discussion in Ben-Yami (2004, pp. 73-75).) However, all three constructions are merged in Fregean logic on its standard interpretation. Already in Frege's Begriffsschrift of 1879, the Aristotelian 'Some S are P' is translated by $\neg \forall \mathrm{x}(\mathrm{Sx} \rightarrow \neg \mathrm{Px})$, which is equivalent to $\neg \forall \mathrm{x} \neg(\mathrm{Sx} \wedge \mathrm{Px})$. (Frege writes these in his two-dimensional script and with different symbols, and he does not use conjunction; but these differences are irrelevant to the points made here.) And earlier in the same Sect. 12, Frege maintains that $\neg \forall \mathrm{x} \neg \mathrm{Qx}$ can be translated, 'There are Q's' ('Es gibt Q's'). Accordingly, Frege here identifies 'Some S are P' with something like, 'There are things which are $\mathrm{S}$ and $\mathrm{P}$ ', and particular quantification is identified with the 'there is' construction. Later, in 1897 , Peano introduced the symbol $\exists$, identifying in the process 'there are A's' and 'A's exist' ('il y a des $a$ ', 'les $a$ existent'; 1897, p. 47). The nineteenth century thus bequeathed us with a formal logic which does not distinguish between particular quantification, instantial sentences, and attribution of existence, an interpretation of the system which became orthodoxy during the first half of the twentieth century.

This is not to say that there is any logical problem with the Begriffsschrift or the Predicate Calculus. Considered as languages in their own right, this merging is legitimate. However, if our formal system aims to represent the logic of Natural Language and of our ordinary concepts, it should distinguish between these three structures. And unlike the Predicate Calculus on its standard interpretation, this is done by Quarc.

We have seen how Quarc incorporates quantification. Existence will be incorporated by a unary predicate, 'E'. As noted, I don't translate the 'there is' construction into Quarc in this paper. Sentences (84) and (85), 'Some Greeks are philosophers' and 'Abraham existed, Adam didn't', are then translated (ignoring tense),

90. $\exists \mathrm{GP}$

91. $b E \wedge d \neg E$

Logically, sentence (90) has no existential import. It does not entail that some Greeks exist, or $\exists \mathrm{GE}$ in Quarc. Although the content or circumstances of the assertion might entail or imply that the Greeks who are philosophers exist, this is not part of the logic of the sentence. The first sentence of example (87), 'Some biblical characters never existed', is therefore translated into Quarc, without any air of paradox in this system, as

\section{2. $\exists \mathrm{B} \neg \mathrm{E}$}

And as was mentioned above, in Quarc as in Natural Language, the particular quantifier is no different than any other quantifier, the universal one included, in having no existential import.

To emphasise the lack of any existential import of particular quantification and to distance it from the predicate 'exist', it would help introducing a new symbol for it into Quarc instead of using the mirror image of ' $E$ ', related to 'Exist'. Using the mirror image of ' $\mathrm{P}$ ' could serve this purpose. But not to burden the reader with new symbols, it is not done in this paper. 
As quantification has no existential import in Quarc, the Quarc sentence which translates 'Necessarily, some Greek is Socrates', $\square(\exists \mathrm{G}=\mathrm{s})$, does not say that Socrates exists, and therefore neither that he exists necessarily. The use of the predicate 'Greek' may imply that we are talking about existing things, but this is not part of the logic of the sentence. Still, it is interesting to see why this Quarc formula, which like the Predicate Calculus' $\square \exists \mathrm{x}(\mathrm{x}=\mathrm{s})$ says of someone-some Greek-that he's necessarily Socrates, is not a theorem:

\begin{tabular}{llll}
\hline & $(1)$ & $\mathrm{s}=\mathrm{s}$ & $=\mathrm{I}$ \\
2 & $(2)$ & $\mathrm{sG}$ & Premise \\
2 & $(3)$ & $\exists \mathrm{G}=\mathrm{s}$ & $\exists \mathrm{I} 1,2$ \\
\hline
\end{tabular}

But now, $\square(\exists \mathrm{G}=\mathrm{s})$ cannot be derived from line (3), since $\exists \mathrm{G}=\mathrm{s}$ is not a theorem, as it depends on the premise sG. Indeed, that some Greek is Socrates is not necessarily true: Socrates might not have been born, and then no Greek would have been Socrates.

However, if we use the predicate Thing, as was done in the previous section, we can prove the following:

\begin{tabular}{lll}
\hline$(1)$ & $\mathrm{s}=\mathrm{s}$ & $=\mathrm{I}$ \\
$(2)$ & $\mathrm{sT}$ & $\mathrm{ThI}$ \\
$(3)$ & $\exists \mathrm{T}=\mathrm{s}$ & $\exists \mathrm{I} 1,2$ \\
$(4)$ & $\square(\exists \mathrm{T}=\mathrm{s})$ & $\mathrm{Nec} 3$ \\
\hline
\end{tabular}

Namely, necessarily, some Thing is Socrates, which can be considered Quarc's translation of the Predicate calculus' $\square \exists x(x=s)$. This conclusion is provable because 'sT' is a theorem. It is valid because of the special interpretation rule of ' $\mathrm{T}$ '; more specifically, unlike 'Greek', it has the same designation at any possibility w, which contains all items designated by any singular argument. (I use 'designation' in this paragraph and the next without defining it, but its exact formal rendering should be clear.) We again see that a theorem of the Predicate Calculus is the result of its specific conception of quantification and does not represent the interaction of quantification and modality generally. And again, this conclusion — that ' $\square(\exists T=s)$ ' is a theorem-is reached by introducing to Quarc a special predicate which does not represent any concept of Natural Language but is used only to approximate the conception of quantification and domain of the Predicate Calculus. From Quarc's point of view, there is no need for such a special predicate apart from for the analysis of quantification in the Predicate Calculus.

It is also relevant to see why the fact that $\square(\exists T=s)$ is a theorem of Quarc does not pose in this system any conceptual problem comparable to Prior's spectre. This is due to the independence, in Quarc, of existence on the one hand, and quantification and reference on the other: ' $\square(\exists \mathrm{T}=\mathrm{s})$ ' does not say or entail that necessarily, Socrates exists; particular quantification has no existential import and the items designated by a singular argument or predicate need not exist. Specifically, to be a Thing, you don't need to exist. 
Our conclusion is therefore that no myth of necessary existence haunts modal Quarc. With 'E' used to predicate existence-the only way in which this concept is captured by Quarc - it has no problematic theorem of the form, $\square \mathrm{sE}, \square(\forall \mathrm{PE})$ or even $\square(\forall \mathrm{TE})$ - this last formula already involving an adaptation to the Predicate Calculus' specific conception of quantification. The problem of necessary existence arises in the Predicate Calculus, on its standard interpretation of $\square \exists x(x=s)$, because it merges three separate Natural Language constructions: particular quantification, predication of existence, and instantial sentences. Once these are distinguished, as they are in Quarc, the problem disappears.

Following this analysis, the Predicate Calculus can be reinterpreted, and its existential quantifier, its name notwithstanding, be considered as having no existential meaning. Its formula (10), $\square \exists x(x=s)$, need not then be interpreted as saying that necessarily, Socrates exists, but only that (i) necessarily, some Thing is Socrates, or that (ii) necessarily, there is a Thing which is Socrates-whether or not that Thing exists. Namely, the conceptual problem posed by such formulas is also a result of their standard yet optional interpretation. Similarly, formula (83), $\square \forall y \square \exists x(x=y)$, is then read, 'Necessarily, every Thing is necessarily some Thing' (Which Thing?-itself), with no implication of existence. This would involve a departure from the canonical interpretation of the Predicate Calculus; however, I think this departure is both acceptable and would draw the meaning of Predicate Calculus formulas closer to Natural Language, although, as noted, quantification over 'Things' does not represent quantification in Natural Language. Moreover, even on this revised Predicate Calculus interpretation, a place for instantial sentences, distinguished from both particular quantification and existence predication, is hard to allocate. One would still tend to see them as an alternative way either of predicating existence (see footnote 8 on Priest) or of particularly quantifying (as was just done in (ii) in this paragraph). Quarc, by contrast, distinguishes these three constructions.

Quarc's separation between, on the one hand, reference and quantification, and on the other, existence, also clarifies why there is no conceptual problem in not relativizing the values of singular terms to possible worlds or possibilities, or why singular terms may refer to the same object relative to all possibilities: reference to an item in a formula and evaluation of the truth-value of that formula relative to a possibility do not presuppose that the item exists at that possibility. Indeed, we need to be able to refer to things also when talking about situations in which they don't exist, as is demonstrated by,

\section{Had Abraham not met Sarah, Isaac wouldn't have existed.}

In this sentence, we are describing a possible situation, one in which Abraham doesn't meet Sarah, and say of Isaac that in that situation he does not exist.

Bearing in mind the observations above, we can also suggest a reinterpretation of the Barcan formulas and their converses in the Predicate Calculus which would make them less problematic. We can then re-examine in its light the apparent counterexamples to them which opened this paper. Since the considerations are similar in both cases, I shall discuss only the apparent counterexample to the converse Barcan formulas. The Predicate Calculus' existential version (3) was,

$$
\exists \mathrm{x} \diamond \phi(\mathrm{x}) \rightarrow \diamond \exists \mathrm{x} \phi(\mathrm{x})
$$


The apparent counterexample to (3) was obtained by interpreting $\phi(\mathrm{x})$ as meaning, $x$ does not exist. We then observed that while the de re antecedent (7), 'There is someone who might not have existed', is true, the de dicto consequent (8), 'It is possible that there be someone who doesn't exist', seems false.

As noted in the previous section, the Quarc approximation of the converse Barcan formula using 'Thing' is valid. In it, the parallel to formula (3) which translates the apparent counterexample, with ' $\mathrm{a} \neg \mathrm{E}$ ' meaning a does not exist, is:

94. $\exists \mathrm{T} \diamond \neg \mathrm{E} \rightarrow \diamond \exists \mathrm{T} \neg \mathrm{E}$

The antecedent and consequent translate the English sentences,

95. Some things might not have existed.

96. It's possible that some things do not exist.

In Quarc, $\exists$ stands for the particular quantifier and has no existential import, and reference is independent of existence; accordingly, both sentences are naturally taken as true. It is possible that Hamlet does not exist, for instance; and since Hamlet is still some Thing, although not an existing thing, $\diamond \exists \mathrm{T} \neg \mathrm{E}$ is true.

This interpretation of Quarc's (94) can now be adapted to the Predicate Calculus, on the lines suggested above, to accommodate formulas (1) to (4). The so-called existential quantifier $\exists$ would then be read as the particular quantifier, with no existential import. As said above, this departure from the canonical interpretation of the Predicate Calculus would draw the meaning of the Predicate Calculus quantifier closer to Natural Language. In addition, following the independence in Quarc of quantification and existence, and similarly of reference and existence, on this semantic reinterpretation of the Predicate Calculus no sort of 'being' should be assigned to the 'things' in the domain. A fixed domain therefore would not suggest a 'possibilist' interpretation of the 'things' in it, according to which merely possible entities have an obscure 'less robust but nonetheless fully-fledged type of being' compared to real things (Menzel 2018; Menzel does not adhere to this position). Rather, we can refer to and quantify over things that do not exist in any sense.

As Garson writes of the Predicate Calculus on its standard model-theoretic interpretation, 'the fixed-domain interpretation has advantages of simplicity and familiarity, but it does not provide a direct account of the semantics of certain quantifier expressions of natural language.' For instance,

on the fixed-domain interpretation, the sentence $\forall y \square \exists x(x=y)$ is valid. Assuming that $\exists x(x=y)$ is read: $y$ exists, $\forall y \square \exists x(x=y)$ says that everything exists necessarily. However, it seems a fundamental feature of common ideas about modality that the existence of many things is contingent, and that different objects exist in different possible worlds. (2016, Sect. 14)

As was argued in previous sections, this and other valid formulas result from a specific, restricted conception of quantification which is hard to justify from a logical point of view. Moreover, as was argued in this section, the standard interpretation renders some of these formulas problematic because of its merge of the concepts of quantification, instantial sentences and existence. If, adopting Quarc's point of view, the semantics is reinterpreted as suggested here, the validity of some of these formulas need not be considered conceptually problematic. 
Quarc disconnects quantification and reference from existence, and quantification in it involves no domain in the sense of the model-theoretic semantics for the Predicate Calculus. The issues mentioned by Garson and Menzel therefore cannot arise in it, and that without any need for a non-standard interpretation. For these and other reasons, merging Modal Propositional Logic with Quarc does not generate the mentioned incompatibility with our common ideas about the contingency of existence. Because of the lack of this and other counterintuitive results, there is no need for a move that would block any formula from being a Quarc theorem, analogues to Kripke's elimination of names from his calculus (1963), a move that artificially impoverished the language and necessitated weakening of the rules for Modal Propositional Logic. Similarly, the motivation to abandon, in face of the difficulty Garson mentions or any other, the rules for quantifiers used in the non-modal version and adopt instead more cumbersome quantifier rules such as those of free logic (see Garson 2016) is eliminated. The naïve expectation, that an unproblematic modal quantified logic can be generated by a straightforward merge of modal propositional logic and non-modal quantified logic, is borne out by Quarc.

Acknowledgements Open access funding provided by Central European University. The research leading to this work was supported by the funding programme, Research Stays for University Academics, 2018 (57381327), of the German Academic Exchange Service (DAAD), and by the Senior Fellowship programme of the Edelstein Center, The Hebrew University of Jerusalem. This funding also enabled me to teach seminars including the material of this paper at the MCMP, LMU Munich and at HUJI, and I'm indebted to all the participants in these seminars. I've also presented part of this material at talks at the Logic and Metaphysics Workshop of CUNY Graduate Center, the Semantics Reading Group of Yale University, and the Philosophy of Mathematics Seminar at Oxford University, and I'm indebted to the audience in these talks. Lastly, this paper significantly improved following comments by the referees of this journal and by Ran Lanzet and Edi Pavlovic.

Open Access This article is licensed under a Creative Commons Attribution 4.0 International License, which permits use, sharing, adaptation, distribution and reproduction in any medium or format, as long as you give appropriate credit to the original author(s) and the source, provide a link to the Creative Commons licence, and indicate if changes were made. The images or other third party material in this article are included in the article's Creative Commons licence, unless indicated otherwise in a credit line to the material. If material is not included in the article's Creative Commons licence and your intended use is not permitted by statutory regulation or exceeds the permitted use, you will need to obtain permission directly from the copyright holder. To view a copy of this licence, visit http://creativecommons.org/licenses/by/4.0/. 


\section{References}

Azzouni, J. (2007). Ontological commitment in the vernacular. Noûs, 41(2), 204-226. https://doi.org/10.1 111/j.1468-0068.2007.00644.x.

Ben-Yami, H. (2004). Logic \& natural language: On plural reference and its semantic and logical significance. London: Routledge.

Ben-Yami, H. (2014). The quantified argument calculus. The Review of Symbolic Logic, 7(1), 120-146. https://doi.org/10.1017/S1755020313000373.

Ben-Yami, H. (Forthcoming). The quantified argument calculus and natural logic. Dialectica.

Ben-Yami, H., \& Pavlovic, E. (Unpublished). The completeness of the quantified argument calculus on the truth-valuational approach.

Crane, T. (2012). Existence and quantification reconsidered. In T. Tahko (Ed.), Aristotelian metaphysics (pp. 44-65). Cambridge: Cambridge University Press.

Crane, T. (2013). The objects of thought. Oxford: Oxford University Press.

Fitch, F. (1952). Symbolic logic. New York, NY: Roland Press.

Fitting, M. (1999). Barcan both ways. Journal of Applied Non-Classical Logics, 9, 329-344. https://doi. org/10.1080/11663081.1999.10510970.

Frege, G. (1879). Begriffsschrift: Eine der Arithmetischen nachgebildete Formelsprache des reinen Denkens. Halle A/S: Verlag von Louis Nebert.

Garson, J. (2016). Modal Logic. In Zalta, E. N. (Ed.) The Stanford Encyclopedia of Philosophy (Spring 2016 Edition). https://plato.stanford.edu/archives/spr2016/entries/logic-modal/.

Geach, P. T. (1962). Reference and generality: An examination of some medieval and modern theories, (emended edition 1968). Ithaca, New York: Cornell University Press.

Jáskowski, S. (1934). On the rules of suppositions in formal logic. Studia Logica, 1, 2-32.

Kripke, S. (1963). Semantical considerations on modal logic. Acta Philosophica Fennica, 16, 83-94.

Lambert, K. (2003). Free logic: Selected essays. Cambridge: Cambridge University Press.

Lanzet, R. (2017). A three-valued quantified argument calculus: Domain-free model-theory, completeness, and embedding of FOL. The Review of Symbolic Logic, 10(3), 549-582. https://doi.org/10.1017/S17 55020317000053.

Lanzet, R., \& Ben-Yami, H. (2004). Logical inquiries into a new formal system with plural reference. In V. F. Hendricks (Ed.), First-order logic revisited (pp. 173-223). Berlin: Logos Verlag.

Lemmon, E. J. (1978). Beginning logic. Indianapolis, London: Hackett.

McGinn, C. (2000). Logical properties: Identity, existence, predication, necessity, truth. Oxford: Oxford University Press.

McNally, L. (1998). Existential sentences without existential quantification. Linguistics and Philosophy, 21(4), 353-392. https://doi.org/10.1023/A:1005389330615.

McNally, L. (2011). Existential Sentences. In C. Maienborn, K. von Heusinger, \& P. Portner (Eds.), Semantics: An international handbook of natural language meaning (pp. 1829-1848). The Hague: de Gruyter.

Menzel, C. (2018). Actualism. In Zalta, E. N. (Ed.) The Stanford Encyclopedia of Philosophy (Summer 2018 Edition). https://plato.stanford.edu/archives/sum2018/entries/actualism/.

Movahed, Z. (2003). Ibn-Sina's anticipation of the formulas of Buridan and Barcan. In Enayat, A., Kalantari, I., \& Moniri, M. (eds). Logic in Teheran: Proceedings of the workshop and conference on logic, algebra and arithmetic, held October 18-22, 2003 (pp. 248-255). LNL 26 Wellesley, MA: ASL/A K Peters, Ltd.

Nolt, J. (2018). Free Logic. In Zalta, E. N. (Ed.) The Stanford Encyclopedia of Philosophy (Fall 2018 Edition). https://plato.stanford.edu/archives/fall2018/entries/logic-free/.

Pavlovic, E. (2017). The quantified argument calculus: An inquiry into its logical properties and applications. PhD thesis, Central European University, Budapest.

Pavlovic, E., \& Gratzl, N. (2019a). Proof-theoretic analysis of the quantified argument calculus. The Review of Symbolic Logic, 12(4), 607-636. https://doi.org/10.1017/S1755020318000114.

Pavlovic, E., \& Gratzl, N. (2019b). Free logic and the quantified argument calculus. In G. M. Mras, P. Weingartner, \& B. Ritter (Eds.), Philosophy of logic and mathematics (pp. 35-45). The Hague: de Gruyter.

Peano, G. (1897). Formulaire de Mathématique. Turin: Bocca Frères, CH. Clausen.

Peters, S., \& Westerståhl, D. (2006). Quantifiers in language and logic. Oxford: Oxford University Press.

Priest, G. (2016). Towards non-being: The logic and metaphysics of intentionality (2nd ed.). Oxford: Oxford University Press. 
Prior, A. (1956). Modality and quantification in S5. Journal of Symbolic Logic, 21(1), 60-62. https://doi. org/10.2307/2268488.

Prior, A. N. (1957). Time and modality. Oxford: Oxford University Press.

Raab, J. (2018). Aristotle, logic, and Quarc. History and Philosophy of Logic, 39(4), 305-340. https://doi. org/10.1080/01445340.2018.1467198.

Rayo, A., \& Uzquiano, G. (Eds.). (2006). Absolute generality. Oxford: Oxford University Press.

Strawson, P. F. (1959). Individuals: An essay in descriptive metaphysics. London: Routledge.

Williamson, T. (2013). Modal logic as metaphysics. Oxford: Oxford University Press.

Yin, H. (Unpublished). Quantified argument calculus: Truth-valuational semantics, completeness, and formal relation to the predicate calculus.

Publisher's Note Springer Nature remains neutral with regard to jurisdictional claims in published maps and institutional affiliations. 